\title{
Centerpole sets for colorings of abelian groups
}

\author{
Taras Banakh • Ostap Chervak
}

Received: 17 March 2010 / Accepted: 15 December 2010 / Published online: 8 January 2011

(C) Springer Science+Business Media, LLC 2011

\begin{abstract}
A subset $C \subset G$ of a group $G$ is called $k$-centerpole if for each $k$-coloring of $G$ there is an infinite monochromatic subset $G$, which is symmetric with respect to a point $c \in C$ in the sense that $S=c S^{-1} c$. By $c_{k}(G)$ we denote the smallest cardinality $c_{k}(G)$ of a $k$-centerpole subset in $G$. We prove that $c_{k}(G)=c_{k}\left(\mathbb{Z}^{m}\right)$ if $G$ is an abelian group of free rank $m \geq k$. Also we prove that $c_{1}\left(\mathbb{Z}^{n+1}\right)=1$, $c_{2}\left(\mathbb{Z}^{n+2}\right)=3, c_{3}\left(\mathbb{Z}^{n+3}\right)=6,8 \leq c_{4}\left(\mathbb{Z}^{n+4}\right) \leq c_{4}\left(\mathbb{Z}^{4}\right)=12$ for all $n \in \omega$, and $\frac{1}{2}\left(k^{2}+3 k-4\right) \leq c_{k}\left(\mathbb{Z}^{n}\right) \leq 2^{k}-1-\max _{s \leq k-2}\left(\begin{array}{c}k-1 \\ s-1\end{array}\right)$ for all $n \geq k \geq 4$.
\end{abstract}

Keywords Abelian group - Centerpole set - Coloring · Symmetric subset . Monochromatic subset

\section{Introduction}

Answering a problem posed in [11], T. Banakh and I. Protasov [4] proved that for any $k$-coloring $\chi: \mathbb{Z}^{k} \rightarrow k=\{0, \ldots, k-1\}$ of the abelian group $\mathbb{Z}^{k}$ there is an infinite monochromatic subset $S \subset \mathbb{Z}^{k}$ such that $S-c=c-S$ for some point $c \in\{0,1\}^{k}$. The equality $S-c=c-S$ means that the set $S$ is symmetric with respect to the point $c$. On the other hand, a suitable partition of $\mathbb{R}^{k}$ into $k+1$ convex cones determines a Borel $(k+1)$-coloring of $\mathbb{R}^{k}$ without unbounded monochromatic symmetric subsets. These two results motivate the following definition, cf. [1, 3].

T. Banakh $(\bowtie) \cdot$ O. Chervak

Ivan Franko National University of Lviv, Lviv, Ukraine

e-mail: t.o.banakh@gmail.com

O. Chervak

e-mail: oschervak@gmail.com

T. Banakh

Uniwersytet Humanistyczno-Przyrodniczy Jana Kochanowskiego, Kielce, Poland 
Definition 1 A subset $C$ of a topological group $G$ is called $k$-centerpole ${ }^{1}$ for (Borel) colorings of $G$ if for any (Borel) $k$-coloring $\chi: G \rightarrow k$ of $G$ there is an unbounded monochromatic subset $S \subset G$, symmetric with respect to some point $c \in C$ in the sense that $S c^{-1}=c S^{-1}$.

The smallest cardinality $|C|$ of such a $k$-centerpole set $C \subset G$ is denoted by $c_{k}(G)$ (resp. $c_{k}^{B}(G)$ ). If no $k$-centerpole set $C \subset G$ exists, then we write $c_{k}(G)=\infty$ (resp. $c_{k}^{B}(G)=\infty$ ) and assume that $\infty$ is greater than any cardinal that appears in our considerations.

Now we explain some terminology that appears in this definition. A subset $B$ of a topological group $G$ is called totally bounded if $B$ can be covered by finitely many left shifts of any neighborhood $U$ of the neutral element of $X$. In the opposite case $B$ is called unbounded. A subset of a discrete topological group is unbounded if and only if it is infinite.

A cardinal number $k$ is identified with the set $\{\alpha:|\alpha|<\kappa\}$ of ordinals of smaller cardinality and endowed with the discrete topology. By a (Borel) $k$-coloring of a topological space $X$ we mean a (Borel) function $\chi: X \rightarrow k$. A function $\chi: X \rightarrow k$ is Borel if for every color $i \in k$ the set $\chi^{-1}(i)$ of points of color $i$ in $X$ is Borel.

The definition of the numbers $c_{k}(G)$ and $c_{k}^{B}(G)$ implies that

$$
c_{k}^{B}(G) \leq c_{k}(G)
$$

for any topological group $G$ and any cardinal number $k$. If the topological group $G$ is discrete, then each coloring of $G$ is Borel, so $c_{k}^{B}(G)=c_{k}(G)$ for all $k$. In general, the cardinal numbers $c_{k}(G)$ and $c_{k}^{B}(G)$ are different. For example, $c_{\omega}^{B}\left(\mathbb{R}^{\omega}\right)=\omega$ while $c_{\omega}\left(\mathbb{R}^{\omega}\right)=\infty$, see Theorem 2 .

It follows from the definition that $c_{k}(G)$ and $c_{k}^{B}(G)$ considered as functions of $k$ and $G$ are non-decreasing with respect to $k$ and non-increasing with respect to $G$. More precisely, for a number $k \in \mathbb{N}$, a topological group $G$ and its subgroup $H$ we have the inequalities

$$
\begin{array}{ll}
c_{k}(H) \geq c_{k}(G), & c_{k}(G) \leq c_{k+1}(G) \quad \text { and } \\
c_{k}^{B}(H) \geq c_{k}^{B}(G), & c_{k}^{B}(G) \leq c_{k+1}^{B}(G) .
\end{array}
$$

In the sequel we shall use these monotonicity properties of $c_{k}(G)$ and $c_{k}^{B}(G)$ without any special reference.

In this paper we investigate the problem of calculating the numbers $c_{k}(G)$ and $c_{k}^{B}(G)$ for an abelian topological group $G$ and show that in many cases this problem reduces to calculating the numbers $c_{k}\left(\mathbb{R}^{n} \times \mathbb{Z}^{m-n}\right)$ and $c_{k}^{B}\left(\mathbb{R}^{n} \times \mathbb{Z}^{m-n}\right)$ where $n=$ $r_{\mathbb{R}}(G)$ is the $\mathbb{R}$-rank and $m=r_{\mathbb{Z}}(G)$ is the $\mathbb{Z}$-rank of the group $G$.

For topological groups $G$ and $H$ the $H$-rank $r_{H}(G)$ of $G$ is defined as

$$
r_{H}(G)=\sup \left\{k \in \omega: H^{k} \hookrightarrow G\right\}
$$

\footnotetext{
${ }^{1}$ So, a centerpole set can be thought as a set of poles of central symmetries that detects unbounded monochromatic symmetric subsets.
} 
where $H^{k} \hookrightarrow G$ means that $H^{k}$ is topologically isomorphic to a subgroup of the topological group $G$. It is clear that $r_{\mathbb{R}}(G) \leq r_{\mathbb{Z}}(G)$ for each topological group $G$.

It is interesting to remark that the $\mathbb{Z}$-rank appears in the formula for calculating the value of the function

$$
\nu(G)=\min \left\{\kappa: c_{k}(G)=\infty\right\}
$$

introduced and studied in [12] and [4]. By [4], for any discrete abelian group $G$

$$
v(G)= \begin{cases}\max \{|G[2]|, \log |G|\} & \text { if } G \text { is uncountable or } G[2] \text { is infinite } \\ r_{\mathbb{Z}}(G)+1 & \text { if } G \text { is finitely generated } \\ r_{\mathbb{Z}}(G)+2 & \text { otherwise. }\end{cases}
$$

Here $G[2]=\{x \in G: 2 x=0\}$ is the Boolean subgroup of $G$ and $\log |G|=\min \{\kappa$ : $\left.|G| \leq 2^{\kappa}\right\}$.

A topological group $G$ is called inductively locally compact (briefly, an ILC-group) if each finitely generated subgroup $H \subset G$ has locally compact closure in $G$. The class of ILC-groups includes all locally compact groups and all closed subgroups of topological vector spaces.

Our aim is to calculate the numbers $c_{k}(G)$ and $c_{k}^{B}(G)$ for an abelian ILC-group $G$. First, let us exclude two cases in which these numbers can be found in a trivial way. One of them happens if the number of colors is 1 . In this case

$$
c_{1}^{B}(G)=c_{1}(G)= \begin{cases}1 & \text { if } G \text { is not totally bounded } \\ \infty & \text { if } G \text { is totally bounded. }\end{cases}
$$

The other trivial case happens if the Boolean subgroup $G[2]=\{x \in G: 2 x=0\} \subset G$ is unbounded in $G$. In this case, for each finite coloring $\chi: G \rightarrow k$ there is a color $i \in k$ such that the set $S=G[2] \cap \chi^{-1}(i)$ is unbounded. Since $S=-S$, we conclude that $S$ is an unbounded monochromatic symmetric subset with respect to 0 , which means that the singleton $\{0\}$ is $k$-centerpole in $G$ and thus

$$
c_{k}(G)=c_{k}^{B}(G)=1 \quad \text { for all } k \in \mathbb{N} \text {. }
$$

It remains to calculate the values of the cardinal numbers $c_{k}(G)$ and $c_{k}^{B}(G)$ for $k \geq 2$ and an abelian topological group $G$ with totally bounded Boolean subgroup $G[2]$.

The following theorem reduces this problem of calculation of $c_{k}(G)$ to the case of the group $\mathbb{R}^{n} \oplus \mathbb{Z}^{m-n}$ where $n=r_{\mathbb{R}}(G)$ and $m=r_{\mathbb{Z}}(G)$.

Theorem 1 Let $k \in \mathbb{N}$ and $G$ be an abelian ILC-group $G$ with totally bounded Boolean subgroup $G[2]$ and ranks $n=r_{\mathbb{R}}(G)$ and $m=r_{\mathbb{Z}}(G)$. Then

(1) $c_{k}(G)=c_{k}\left(\mathbb{R}^{n} \times \mathbb{Z}^{m-n}\right)$ if $k \leq m$, and

(2) $c_{k}(G) \geq \omega$ if $k>m$.

If the topological group $G$ is metrizable, then 
(3) $c_{k}^{B}(G)=c_{k}^{B}\left(\mathbb{R}^{n} \times \mathbb{Z}^{m-n}\right)$ if $k \leq m$, and

(4) $c_{k}^{B}(G) \geq \omega$ if $k>m$.

Here we assume that $\omega-\omega=0$ and $\omega-n=\omega$ for each $n \in \omega$.

Theorem 1 will be proved in Sect. 10. It reduces the problem of calculation of the numbers $c_{k}(G)$ and $c_{k}^{B}(G)$ to calculating these numbers for the groups $\mathbb{R}^{n} \times \mathbb{Z}^{m-n}$ where $n \leq m$. The latter problem turned out to be highly non-trivial. In the following theorem we collect all the available information on the precise values of the numbers $c_{k}\left(\mathbb{R}^{n} \times \mathbb{Z}^{m}\right)$ and $c_{k}^{B}\left(\mathbb{R}^{n} \times \mathbb{Z}^{m}\right)$.

Theorem 2 Let $k, n, m$ be cardinal numbers.

(1) If $n+m \geq 1$, then $c_{1}^{B}\left(\mathbb{R}^{n} \times \mathbb{Z}^{m}\right)=c_{1}\left(\mathbb{R}^{n} \times \mathbb{Z}^{m}\right)=1$.

(2) If $n+m \geq 2$, then $c_{2}^{B}\left(\mathbb{R}^{n} \times \mathbb{Z}^{m}\right)=c_{2}\left(\mathbb{R}^{n} \times \mathbb{Z}^{m}\right)=3$.

(3) If $n+m \geq 3$, then $c_{3}^{B}\left(\mathbb{R}^{n} \times \mathbb{Z}^{m}\right)=c_{3}\left(\mathbb{R}^{n} \times \mathbb{Z}^{m}\right)=6$.

(4) If $n+m=4$, then $c_{4}^{B}\left(\mathbb{R}^{n} \times \mathbb{Z}^{m}\right)=c_{4}\left(\mathbb{R}^{n} \times \mathbb{Z}^{m}\right)=12$.

(5) If $k \geq n+m+1<\omega$, then $c_{k}^{B}\left(\mathbb{R}^{n} \times \mathbb{Z}^{m}\right)=\infty$.

(6) If $k \geq n+m+1$, then $c_{k}\left(\mathbb{R}^{n} \times \mathbb{Z}^{m}\right)=\infty$.

(7) If $n+m \geq \omega$ and $\omega \leq k<\operatorname{cov}(\mathcal{M})$, then $c_{k}^{B}\left(\mathbb{R}^{n} \times \mathbb{Z}^{m}\right)=\omega$.

In the last item by $\operatorname{cov}(\mathcal{M})$ we denote the smallest cardinality of the cover of the real line by meager subsets. It is known that $\aleph_{1} \leq \operatorname{cov}(\mathcal{M}) \leq \mathfrak{c}$ and the equality $\operatorname{cov}(\mathcal{M})=\mathfrak{c}$ is equivalent to the Martin Axiom for countable posets, see [9, 19.9].

The equality $c_{4}\left(\mathbb{Z}^{4}\right)=12$ from the statement (4) of Theorem 2 answers the problem of the calculation of $c_{4}\left(\mathbb{Z}^{4}\right)$ posed in [1] and then repeated in [5, Problem 2.4], [6, Problem 12], and [2, Question 4.5].

Theorem 2 presents all cases in which the exact values of the cardinals $c_{k}^{B}\left(\mathbb{R}^{n} \times\right.$ $\left.\mathbb{Z}^{m-n}\right)$ and $c_{k}\left(\mathbb{R}^{n} \times \mathbb{Z}^{m-n}\right)$ are known. In the remaining cases we have some upper and lower bounds for these numbers. Because of the inequalities

$$
c_{k}^{B}\left(\mathbb{R}^{m}\right) \leq c_{k}^{B}\left(\mathbb{R}^{n} \times \mathbb{Z}^{m-n}\right) \leq c_{k}\left(\mathbb{R}^{n} \times \mathbb{Z}^{m-n}\right) \leq c_{k}\left(\mathbb{Z}^{m}\right),
$$

we see that the upper bounds for the numbers $c_{k}^{B}\left(\mathbb{R}^{n} \times \mathbb{Z}^{m-n}\right)$ and $c_{k}\left(\mathbb{R}^{n} \times \mathbb{Z}^{m-n}\right)$ would follow from the upper bounds for the numbers $c_{k}\left(\mathbb{Z}^{m}\right)$ while lower bounds from lower bounds on $c_{k}^{B}\left(\mathbb{R}^{m}\right)$.

Theorem 3 For any numbers $k \in \mathbb{N}$ and $n, m \in \mathbb{N} \cup\{\omega\}$, we get:

(1) $c_{k}\left(\mathbb{Z}^{m}\right) \leq 2^{k}-1-\max _{s \leq k-2}\left(\begin{array}{l}k-1 \\ s-1\end{array}\right)$ if $k \leq m$,

(2) $c_{k}^{B}\left(\mathbb{R}^{k}\right) \geq \frac{1}{2}\left(k^{2}+3 k-4\right)$ if $k \geq 4$,

(3) $c_{k}^{B}\left(\mathbb{R}^{m}\right) \geq k+4$ if $m \geq k \geq 4$,

(4) $c_{k}^{B}\left(\mathbb{R}^{n}\right)<c_{k+1}^{B}\left(\mathbb{R}^{n+1}\right)$ and $c_{k}\left(\mathbb{R}^{n}\right)<c_{k+1}\left(\mathbb{R}^{n+1}\right)$ if $k \leq n$,

(5) $c_{k}^{B}\left(\mathbb{R}^{n} \times \mathbb{Z}^{m}\right)<c_{k+1}^{B}\left(\mathbb{R}^{n} \times \mathbb{Z}^{m+1}\right)$ and $c_{k}\left(\mathbb{R}^{n} \times \mathbb{Z}^{m}\right)<c_{k+1}\left(\mathbb{R}^{n} \times \mathbb{Z}^{m+1}\right)$ if $k \leq$ $n+m$.

The binomial coefficient $\left(\begin{array}{l}k \\ i\end{array}\right)$ in statement (1) equals $\frac{k !}{i !(k-i) !}$ if $i \in\{0, \ldots, k\}$ and zero otherwise. The upper bound from this statement improves the previously known 
upper bound $c_{k}\left(\mathbb{Z}^{n}\right) \leq 2^{k}-1$ proved in [1]. For $k=m \leq 4$ it yields the upper bounds which coincide with the values of $c_{k}\left(\mathbb{Z}^{m}\right)$ given in Theorem 2 .

The lower bound $c_{n}^{B}\left(\mathbb{R}^{n}\right) \geq \frac{1}{2}\left(n^{2}+3 n-4\right)$ from the item (2) improves the previously known lower bound $c_{n}^{B}\left(\mathbb{R}^{n}\right) \geq \frac{1}{2}\left(n^{2}+n\right)$, proved in [1]. For $n=4$ it gives the lower bound $12 \leq c_{4}^{B}\left(\mathbb{R}^{4}\right)$, which coincides with the value of $c_{4}^{B}\left(\mathbb{R}^{4}\right)=c_{4}\left(\mathbb{Z}^{4}\right)$.

The statement (5) implies that the sequence $\left(c_{k}\left(\mathbb{Z}^{k}\right)\right)_{k=1}^{\infty}$ is strictly increasing, which answers Question 2 posed in [1]. Theorem 3 will be proved in Sect. 8 after some preparatory work done in Sect. 2.

For every $k \in \mathbb{N}$ the sequence $\left(c_{k}\left(\mathbb{Z}^{n}\right)\right)_{n=k}^{\infty}$ is non-increasing and thus it stabilizes starting from some $n$. The value of this number $n$ is upper bounded by the cardinal number $r c_{k}^{B}\left(\mathbb{Z}^{n}\right)$ defined as follows.

For a topological group $G$ and a number $k \in \mathbb{N}$ let $r c_{k}^{B}(G)$ be the minimal possible $\mathbb{Z}$-rank $r_{\mathbb{Z}}(\langle C\rangle)$ of a subgroup $\langle C\rangle$ of $G$ generated by a $k$-centerpole subset $C \subset G$ of cardinality $|C|=c_{k}^{B}(G)$. If such a set $C$ does not exist (which happens if $c_{k}^{B}(G)=$ $\infty)$, then we put $r c_{k}^{B}(G)=\infty$.

Theorem 4 (Stabilization) Let $k \geq 2$ be an integer and $G$ be an abelian ILC-group with totally bounded Boolean subgroup $G[2]$ and $\mathbb{R}$-rank $n=r_{\mathbb{R}}(G)$. Then

(1) $c_{k}(G)=c_{k}^{B}\left(\mathbb{Z}^{\omega}\right)$ if $r_{\mathbb{Z}}(G) \geq r c_{k}^{B}\left(\mathbb{Z}^{\omega}\right)$,

(2) $c_{k}^{B}(G)=c_{k}^{B}\left(\mathbb{R}^{n} \times \mathbb{Z}^{\omega}\right)$ if $G$ is metrizable and $r_{\mathbb{Z}}(G) \geq r c_{k}^{B}\left(\mathbb{R}^{n} \times \mathbb{Z}^{\omega}\right)$,

(3) $c_{k}^{B}(G)=c_{k}^{B}\left(\mathbb{R}^{\omega}\right)$ if $G$ is metrizable and $r_{\mathbb{R}}(\mathbb{R}) \geq r c_{k}^{B}\left(\mathbb{R}^{\omega}\right)$.

In light of Theorem 4 it is important to have lower and upper bounds for the numbers $r c_{k}(G)$.

Proposition 1 For any metrizable abelian ILC-group $G$ with totally bounded Boolean subgroup $G[2]$, and a natural number $2 \leq k \leq r_{\mathbb{Z}}(G)$ we get

(1) $r c_{k}^{B}(G)=k$ if $k \leq 3$, and

(2) $k \leq r c_{k}^{B}(G) \leq c_{k}^{B}(G)-3$ if $k \geq 3$.

Finally, let us present the $(k+1)$-centerpole subset $\Xi_{s}^{k}$ of $\mathbb{R}^{1+k}$ that contains $2^{k}-1-\left(\begin{array}{l}k \\ s\end{array}\right)$ elements and gives the upper bound from Theorem 3(1). This $(k+1)$ centerpole set $\Xi_{k}$ is called the $\left(\begin{array}{l}k \\ s\end{array}\right)$-sandwich.

Definition 2 Let $k$ be a non-negative integer and $s$ be a real number. The subsets

$$
\mathbf{2}_{<s}^{k}=\left\{\left(x_{i}\right) \in \mathbf{2}^{k}: \sum_{i=1}^{k} x_{i}<s\right\} \quad \text { and } \quad \mathbf{2}_{>s}^{k}=\left\{\left(x_{i}\right) \in \mathbf{2}^{k}: \sum_{i=1}^{k} x_{i}>s\right\}
$$

are called the $s$-slices of the $k$-cube $\mathbf{2}^{k}$ where $\mathbf{2}=\{0,1\}$ is the doubleton. For $s \in$ $\{0, \ldots, k\}$ the union of such slices has cardinality

$$
\left|2_{<s}^{k} \cup 2_{>s}^{k}\right|=2^{k}-\left(\begin{array}{l}
k \\
s
\end{array}\right)=2^{k}-\frac{k !}{s !(k-s) !} .
$$


The subset

$$
\boldsymbol{\Xi}_{s}^{k}=\left(\{-1\} \times \mathbf{2}_{<s}^{k}\right) \cup\left(\{0\} \times \mathbf{2}_{<k}^{k}\right) \cup\left(\{1\} \times \mathbf{2}_{>S}^{k}\right)
$$

of the group $\mathbb{Z} \times \mathbb{Z}^{k}$ is called the $\left(\begin{array}{l}k \\ s\end{array}\right)$-sandwich. For $s \in\{0, \ldots, k\}$ it has cardinality

$$
\left|\Xi_{s}^{k}\right|=\left|\mathbf{2}_{<k}^{k}\right| \cup\left|\mathbf{2}_{<s}^{k} \cup \mathbf{2}_{>s}^{k}\right|=2^{k+1}-1-\left(\begin{array}{l}
k \\
s
\end{array}\right) \text {. }
$$

The following theorem implies the upper bound in Theorem 3(1). The proof of this theorem (given in Sect. 3) is not trivial and uses some elements of Algebraic Topology.

Theorem 5 For every $k \in \mathbb{N}$ and $s \leq k-2$ the $\left(\begin{array}{l}k \\ s\end{array}\right)$-sandwich $\Xi_{s}^{k}$ is a $(k+1)$ centerpole set in the group $\mathbb{Z} \times \mathbb{Z}^{k}$.

In light of this theorem it is important to know the geometric structure of $\left(\begin{array}{l}k \\ s\end{array}\right)$ sandwiches $\Xi_{s}^{k}$ for $s \leq k-2$. For $k \leq 3$ those sandwiches are written below:

- $\Xi_{-2}^{0}=\{(1,0)\}$ is a singleton in $\mathbb{Z} \times \mathbb{Z}^{0}=\mathbb{Z} \times\{0\}$;

- $\Xi_{-1}^{1}=\{(0,1),(1,0),(1,1)\}$ is the unit square without a vertex in $\mathbb{Z}^{2}$;

- $\Xi_{0}^{2}=\{(0,0,0),(0,0,1),(0,1,0),(1,0,1),(1,1,0),(1,1,1)\}$ is the unit cube without two opposite vertices in $\mathbb{Z}^{3}$;

- $\Xi_{0}^{3}$ is the unit cube without two opposite vertices in $\mathbb{Z}^{4}$, so $\left|\Xi_{0}^{3}\right|=14$;

- $\boldsymbol{\Xi}_{1}^{3}$ is a 12-element subset in $\mathbb{Z}^{4}$ whose slices $\{-1\} \times \mathbf{2}_{<1}^{3},\{0\} \times \mathbf{2}_{<3}^{3}$, and $\{1\} \times \mathbf{2}_{>1}^{3}$ have one, seven, and four points, respectively.

By a triangle (centered at the origin) we shall understand any affinely independent subset $\{a, b, c\}$ in $\mathbb{R}^{n}$ (such that $a+b+c=0$ ). A tetrahedron (centered at the origin) is any affinely independent subset $\{a, b, c, d\} \subset \mathbb{R}^{n}$ (with $a+b+c+d=0$ ).

Let us observe that the sandwich

- $\Xi_{-2}^{0}$ has cardinality $c_{1}\left(\mathbb{R}^{1}\right)=1$ and is affinely equivalent to any singleton $\{a\}$ in $\mathbb{R}^{1}$;

- $\Xi_{-1}^{1}$ has cardinality $c_{2}\left(\mathbb{R}^{2}\right)=3$ and is affinely equivalent to any triangle $\Delta=$ $\{a, b, c\}$ in $\mathbb{R}^{2}$;

- $\Xi_{0}^{2}$ has cardinality $c_{3}\left(\mathbb{R}^{3}\right)=6$ and is affinely equivalent to $\Delta \cup(x-\Delta)$ where $\Delta \subset \mathbb{R}^{3}$ is a triangle centered at zero and $x \in \mathbb{R}^{3}$ does not belong to the linear span of $\Delta$;

- $\Xi_{1}^{3}$ has cardinality $c_{4}\left(\mathbb{R}^{4}\right)=12$ and is affinely equivalent to $(x-\Delta) \cup \Delta \cup(-x-$ $\Delta$ ) where $\Delta \subset \mathbb{R}^{4}$ is a tetrahedron centered at zero and $x \in \mathbb{R}^{4}$ does not belong to the linear span of $\Delta$.

To see that $\Xi_{1}^{3}$ is of this form, observe that $c=\left(\frac{1}{4}, \frac{1}{2}, \frac{1}{2}, \frac{1}{2}\right)$ is the barycenter of $\Xi_{1}^{3}$ and $\Xi_{1}^{3}-c=(x-\Delta) \cup \Delta \cup(-x-\Delta)$ for the tetrahedron

$$
\Delta=\{(0,0,0,1),(0,0,1,0),(0,1,0,0),(1,1,1,1)\}-c
$$

and the point $x=\left(\frac{1}{2}, 0,0,0\right)$. 
Now we briefly describe the structure of this paper. In Sect. 2 we establish a covering property of sandwiches, which will be essentially used in the proof of Theorem 5 , given in Sect. 3. Section 4 is devoted to T-shaped sets which will give us lower bounds for the numbers $c_{k}^{B}\left(\mathbb{R}^{k}\right)$. In Sect. 5 we prove some lemmas that will help us to analyze the geometric structure of centerpole sets in Euclidean spaces. In Sect. 6 we study the interplay between centerpole properties of subsets in a group and those of its subgroups. In Sect. 7 we prove a particular case of the Stability Theorem 4 for the groups $\mathbb{R}^{n} \times \mathbb{Z}^{m-n}$. In Sects. 8, 9, and 10 we give the proofs of Theorems 3, 2, and 1, respectively. Sections 11 and 12 are devoted to the proofs of Proposition 1 and Theorem 4. The final Sect. 13 contains selected open problems.

\section{Covering $\Sigma_{0}$-sets by shifts of the sandwich $\Xi_{s}^{k}$}

In this section we shall prove a crucial covering property of the $\left(\begin{array}{l}k \\ s\end{array}\right)$-sandwich $\Xi_{s}^{k}$. In the next section this property will be used in the proof of Theorem 5. We assume that $k \in \omega$ and $s \leq k-2$ is an integer.

First we introduce the notion of a $\Sigma_{0}$-subset of the cube $2^{k+1}=\{0,1\}^{k+1}$. For $i \in\{0, \ldots, k\}$ consider the $i$ th coordinate projection

$$
\operatorname{pr}_{i}: \mathbb{R}^{k+1} \rightarrow \mathbb{R}, \quad \operatorname{pr}_{i}:\left(x_{j}\right)_{j=0}^{k} \mapsto x_{i}
$$

The subsets of the form $2^{k+1} \cap \operatorname{pr}_{i}^{-1}(l)$ for $l \in\{0,1\}$ are called the facets of the cube $2^{k+1}$.

Next, consider the function

$$
\Sigma: \mathbb{R}^{k+1} \rightarrow \mathbb{R}, \quad \Sigma:\left(x_{i}\right)_{i=0}^{k} \mapsto \sum_{i=1}^{k} x_{i}
$$

and observe that $\Sigma\left(\mathbf{2}^{k+1}\right)=\{0, \ldots, k\}$.

Taking the diagonal product of the functions $\operatorname{pr}_{0}$ and $\Sigma$, we obtain the linear operator

$$
\Sigma_{0}: \mathbb{R}^{k+1} \rightarrow \mathbb{R}^{2}, \quad \Sigma_{0}:\left(x_{i}\right)_{i=0}^{k} \mapsto\left(x_{0}, \sum_{i=1}^{k} x_{i}\right)
$$

Definition 3 A subset $\tau \subset 2^{k+1}$ will be called a $\Sigma_{0}$-set if

- $\tau$ lies in a facet of $\mathbf{2}^{k+1}$;

- there exists $a \in\{0, \ldots, k-1\}$ such that $\Sigma_{0}(\tau) \subset\{(0, a),(0, a+1),(1, a+1)\}$ or $\Sigma_{0}(\tau) \subset\{(0, a),(1, a),(1, a+1)\}$.

Lemma 1 Each $\Sigma_{0}$-set $\tau \subset \mathbf{2}^{k+1}$ is covered by a suitable shift $x+\Xi_{s}^{k}$ of the $\left(\begin{array}{l}k \\ s\end{array}\right)$ sandwich $\Xi_{s}^{k}$. 
Proof Decompose the $\Sigma_{0}$-set $\tau$ into the union $\tau=\tau_{0} \cup \tau_{1}$ where $\tau_{i}=\tau \cap \operatorname{pr}_{0}^{-1}(i)$ for $i \in\{0,1\}$. By our hypothesis $\tau$ lies in a facet of the cube $2^{k+1}$. Consequently, there are numbers $\gamma \in\{0, \ldots, k\}$ and $l \in\{0,1\}$ such that $\tau \subset \operatorname{pr}_{\gamma}^{-1}(l)$. If $\tau_{0}$ or $\tau_{1}$ is empty, then we can change the facet and assume that $\gamma=0$.

Since $\tau$ is a $\Sigma_{0}$-set, the image $\Sigma_{0}(\tau)$ lies in one of the triangles: $\{(0, a),(0, a+1)$, $(1, a+1)\}$ or $(\{(0, a),(1, a),(1, a+1)\}$ for some $a \in\{0, \ldots, k-1\}$. This implies that $\Sigma(\tau) \subset\{a, a+1\}$.

Identify the cube $2^{k}$ with the subcube $\{0\} \times 2^{k}$ of $\Xi_{s}^{k}$ and let $\mathbf{e}_{0}=(1,0, \ldots, 0) \in$ $2^{k+1}$. Then

$$
\Xi_{s}^{k}=\mathbf{2}_{<k}^{k} \cup\left(\mathbf{e}_{0}+\mathbf{2}_{>S}^{k}\right) \cup\left(-\mathbf{e}_{0}+\mathbf{2}_{<s}^{k}\right) .
$$

Depending on the value of $\gamma$, two cases are possible.

0. $\gamma=0$. This case has four subcases.

0.1. If $l=0$ and $a<k-1$ then $\Sigma_{0}(\tau) \subset\{(0, a),(0, a+1)\} \subset\{0, \ldots, k-1\}$ and $\tau \subset 2_{<k}^{k} \subset \Xi_{s}^{k}$.

0.2. If $l=0$ and $a \geq k-1$, then $a>k-2 \geq s$ and $\tau \subset \mathbf{2}_{>s}^{k} \subset-\mathbf{e}_{0}+\boldsymbol{\Xi}_{s}^{k}$.

0.3. If $l=1$ and $a<k-1$, then $\Sigma_{0}(\tau) \subset\{(1, a),(1, a+1)\} \subset\{0, \ldots, k-1\}$ and hence $\tau \subset \mathbf{e}_{0}+\mathbf{2}_{<k}^{k} \subset \mathbf{e}_{0}+\Xi_{s}^{k}$.

0.4. If $l=1$ and $a \geq k-1$, then $a>k-2 \geq s$ and then $\tau \subset \mathbf{e}_{0}+2_{>s}^{k} \subset \Xi_{s}^{k}$.

I. $\gamma \neq 0$. In this case $\tau_{0}$ and $\tau_{1}$ are not empty. Let $\mathbf{e}_{\gamma}$ be the basic vector whose $\gamma$ th coordinate is 1 and the others are zero. By our assumption, $\Sigma_{0}(\tau) \subset$ $\{(0, a),(1, a),(1, a+1)\}$ or $\Sigma_{0}(\tau) \subset\{(0, a),(0, a+1),(1, a+1)\}$ for some $a \in\{0, \ldots, k-1\}$. So, we consider two subcases.

I.1. $\Sigma_{0}(\tau) \subset\{(0, a),(1, a),(1, a+1)\}$. This case has two subcases.

I.1.0. $l=0$. In this subcase $\Sigma(\tau)=\Sigma\left(\tau_{0}\right) \cup \Sigma\left(\tau_{1}\right)=\{a, a+1\} \subset\{0, \ldots$, $k-1\}$ and hence $a \leq k-2$. Depending on the value of $a$, we have three possibilities.

If $a>s$, then $\tau=\tau_{0} \cup \tau_{1} \subset \mathbf{2}_{<k}^{k} \cup\left(\mathbf{e}_{0}+\mathbf{2}_{>s}^{k}\right) \subset \boldsymbol{\Xi}_{s}^{k}$.

If $a=s$, then for the shifted set $\mathbf{e}_{\gamma}+\tau$ we get

$$
\Sigma_{0}\left(\mathbf{e}_{\gamma}+\tau\right) \subset\{(0, a+1),(1, a+1),(1, a+2)\} .
$$

Since $a=s \leq k-2$, we conclude that $\mathbf{e}_{\gamma}+\tau_{0} \subset \mathbf{2}_{<k}^{k} \subset \boldsymbol{\Xi}_{s}^{k}$. On the other hand, $\mathbf{e}_{\gamma}+\tau_{1} \subset \mathbf{e}_{1}+\mathbf{2}_{>s}^{k} \subset \boldsymbol{\Xi}_{s}^{k}$. Then $\tau \subset-\mathbf{e}_{\gamma}+\Xi_{s}^{k}$.

If $a<s$, then $a+1 \leq s \leq k-2$ and hence $\tau=\tau_{0} \cup \tau_{1} \subset \mathbf{2}_{<s}^{k} \cup\left(\mathbf{e}_{0}+\right.$ $\left.\mathbf{2}_{<k}^{k}\right) \subset \mathbf{e}_{0}+\Xi_{s}^{k}$.

I.1.1. $l=1$. In this subcase three possibilities can occur:

If $a>s$, then $\tau=\tau_{0} \cup \tau_{1} \subset \mathbf{2}_{<k}^{k}+\left(\mathbf{e}_{0}+\mathbf{2}_{>s}^{k}\right) \subset \boldsymbol{\Xi}_{s}^{k}$;

If $a<s$, then $a+1 \leq s \leq k-2$ and then $\tau=\tau_{0} \cup \tau_{1} \subset \mathbf{2}_{<s}^{k} \cup\left(\mathbf{e}_{0}+\right.$ $\left.\mathbf{2}_{<k}^{k}\right) \subset \mathbf{e}_{0}+\Xi_{s}^{k}$.

If $a=s$, then for the shift $-\mathbf{e}_{\gamma}+\tau$ we get $\Sigma_{0}\left(-\mathbf{e}_{\gamma}+\tau\right) \subset$ $\{(0, a-1),(1, a-1),(1, a)\}$ and hence $-\mathbf{e}_{\gamma}+\tau \subset \mathbf{2}_{<s}^{k} \cup\left(\mathbf{e}_{0}+\mathbf{2}_{<k}^{k}\right) \subset$ $\mathbf{e}_{0}+\Xi_{s}^{k}$. Consequently, $\tau \subset \mathbf{e}_{\gamma}+\mathbf{e}_{0}+\Xi_{s}^{k}$.

I.2. $\Sigma_{0}(\tau) \subset\{(0, a),(0, a+1),(1, a+1)\}$. Depending on the value of $l \in\{0,1\}$, consider two subcases. 
I.2.0. $l=0$. In this case $\{0, \ldots, k-1\} \supset \Sigma(\tau)=\Sigma\left(\tau_{0}\right) \cup \Sigma\left(\tau_{1}\right)=\{a$, $a+1\} \cup\{a+1\}$ and consequently, $a+1 \leq k-1$.

If $a \geq s$, then $\tau=\tau_{0} \cup \tau_{1} \subset \mathbf{2}_{<k}^{k} \cup\left(\mathbf{e}_{0}+\mathbf{2}_{>s}^{k}\right) \subset \boldsymbol{\Xi}_{s}^{k}$.

If $a=s-1$, then we can consider the shift $\mathbf{e}_{\gamma}+\tau$ and repeating the preceding argument, show that $\mathbf{e}_{\gamma}+\tau \subset \Xi_{s}^{k}$. Consequently, $\tau \subset$ $-\mathbf{e}_{\gamma}+\Xi_{s}^{k}$.

If $a<s-1$, then $\tau=\tau_{0} \cup \tau_{1} \subset \mathbf{2}_{<s}^{k} \cup\left(\mathbf{e}_{0}+\mathbf{2}_{<k}^{k}\right) \subset \mathbf{e}_{0}+\Xi_{s}^{k}$.

I.2.1. $l=1$. In this case we have four subcases.

If $a=k-1$, then for the shifted set $-\mathbf{e}_{\gamma}+\tau$ we get $\Sigma_{0}\left(-\mathbf{e}_{\gamma}+\tau\right) \subset$ $\{(0, a-1),(0, a),(1, a)\}$ and $-\mathbf{e}_{\gamma}+\tau \subset \mathbf{2}_{<k}^{k} \cup\left(\mathbf{e}_{0}+\mathbf{2}_{>s}^{k}\right)=\boldsymbol{\Xi}_{s}^{k}$. Then $\tau \subset \mathbf{e}_{\gamma}+\Xi_{s}^{k}$.

If $s \leq a<k-1$, then $\tau=\tau_{0} \cup \tau_{1} \subset \mathbf{2}_{<k}^{k} \cup\left(\mathbf{e}_{0}+\mathbf{2}_{>s}^{k}\right)=\Xi_{s}^{k}$.

If $a=s-1$, then for the shifted set $-\mathbf{e}_{\gamma}+\tau$ we get $\Sigma_{0}\left(-\mathbf{e}_{\gamma}+\tau\right) \subset$ $\{(0, a-1),(0, a),(1, a)\}$ and then $-\mathbf{e}_{\gamma}+\tau \subset 2_{<s}^{k} \cup\left(\mathbf{e}_{0}+\mathbf{2}_{<k}^{k}\right)=\mathbf{e}_{0}+$ $\Xi_{s}^{k}$ and $\tau \subset \mathbf{e}_{\gamma}+\mathbf{e}_{0}+\Xi_{s}^{k}$.

If $a<s-1$, then $\tau=\tau_{0} \cup \tau_{1} \subset \mathbf{2}_{<s}^{k} \cup\left(\mathbf{e}_{0}+\mathbf{2}_{<k}^{k}\right)=\mathbf{e}_{0}+\Xi_{s}^{k}$.

This was the last of the 17 cases we have considered.

\section{Proof of Theorem 5}

The proof of Theorem 5 uses the idea of the proof of Lemma 6 in [1] (which established the upper bound $\left.c_{3}\left(\mathbb{Z}^{3}\right) \leq 6\right)$.

We need to prove that for every $k \leq n$ and $s \leq k-2$ the $\left(\begin{array}{l}k \\ s\end{array}\right)$-sandwich $\Xi_{s}^{k}$ is $(k+1)$-centerpole in $\mathbb{Z} \times \mathbb{Z}^{k}=\mathbb{Z}^{1+k}$. Assuming that this is not true, find a coloring $\chi$ : $\mathbb{Z}^{1+k} \rightarrow k+1=\{0, \ldots, k\}$ such that $\mathbb{Z}^{1+k}$ contains no unbounded monochromatic subset, symmetric with respect to some point $c \in \Xi_{s}^{k}$. Observe that for each color $i \in\{0, \ldots, k\}$ the intersection $A_{i} \cap\left(2 c-A_{i}\right)$ is the largest subset of $A_{i}$, symmetric with respect to the point $c$. By our assumption, the (maximal $i$-colored $c$-symmetric) set $A_{i} \cap\left(2 c-A_{i}\right)$ is bounded and so is the union

$$
B=\bigcup_{i=0}^{k} \bigcup_{c \in \Xi_{s}^{k}} A_{i} \cap\left(2 c-A_{i}\right)
$$

of all such maximal symmetric monochromatic subsets.

Claim $1 \chi(x) \notin \chi\left(-x+2 \Xi_{s}^{k}\right)$ for any $x \notin B$.

Proof Assuming conversely that $\chi(x)=\chi(-x+2 c)$ for some $c \in \Xi_{s}^{k}$, we get $\frac{1}{2}(x+(-x+2 c))=c$ and hence $x$ and $-x+2 c$ are two points symmetric with respect to the center $c \in \Xi_{s}^{k}$ and colored by the same color. Consequently, $x \in B$ by the definition of $B$.

Fix a number $n \in \mathbb{N}$ so big that the cube $K=[-2 n, 2 n]^{1+k} \subset \mathbb{R}^{1+k}$ contains the bounded set $B$ in its interior and let $\partial K$ be the topological boundary $\partial K$ of the cube $K$ in $\mathbb{R}^{1+k}$. Observe that Claim 1 implies: 
Claim $2 \chi(-x) \notin \chi\left(x+2 \Xi_{s}^{k}\right)$ for each point $x \in \mathbb{Z}^{1+k} \cap \partial K$.

We recall that for every $i \in k+1=\{0, \ldots, k\}$

$$
\operatorname{pr}_{i}: \mathbb{R}^{1+k} \rightarrow \mathbb{R}, \quad \operatorname{pr}_{i}:\left(x_{j}\right)_{j=0}^{k} \mapsto x_{i},
$$

denotes the $i$ th coordinate projection and $\mathbf{e}_{i}$ is the unit vector along the $i$ th coordinate axis, that is, $\operatorname{pr}_{j}\left(\mathbf{e}_{i}\right)=1$ if $i=j$, and 0 otherwise.

For a subset $J \subset\{0, \ldots, k\}$ let $\mathbf{e}_{J}=\sum_{j \in J} \mathbf{e}_{j} \in \mathbb{R}^{1+k}$ be the vector of the principal diagonal of the cube $\mathbf{2}^{J}=\left\{\left(x_{i}\right)_{i=0}^{k} \in \mathbf{2}^{1+k}: \forall i \notin J\left(x_{i}=0\right)\right\} \subset \mathbf{2}^{1+k}$.

For a point $x \in \mathbb{R}^{1+k}$ let $J_{x}=\left\{i \in k+1: x_{i} \notin 2 \mathbb{Z}\right\}$ and let $\lfloor x\rfloor$ be the unique point in $(2 \mathbb{Z})^{1+k}$ such that $x \in\lfloor x\rfloor+2 \cdot 2^{J_{x}}$. So, $\lfloor x\rfloor \leq x \leq\lfloor x\rfloor+2 \mathbf{e}_{J_{x}}$.

Consider the function $\Sigma: \mathbb{R}^{k+1} \rightarrow \mathbb{R}$ assigning to each sequence $x=\left(x_{i}\right)_{i=0}^{k}$ the sum $\Sigma(x)=\sum_{i=1}^{k} x_{i}$. The map $\Sigma$ combined with the 0th coordinate projection $\mathrm{pr}_{0}$ compose the linear operator

$$
\Sigma_{0}: \mathbb{R}^{1+k} \rightarrow \mathbb{R}^{2}, \quad \Sigma_{0}:\left(x_{i}\right)_{i=0}^{k} \mapsto\left(x_{0}, \Sigma(x)\right)=\left(x_{0}, \sum_{i=1}^{k} x_{i}\right) .
$$

Choose a triangulation $T$ of the boundary $\partial K$ of the cube $K=[-2 n, 2 n]^{1+k}$ such that for each simplex $\tau$ of the triangulation there is a point $\dot{\tau} \in(2 \mathbb{Z})^{1+k}$ such that $\frac{1}{2}(\tau-\dot{\tau})$ is a $\Sigma_{0}$-subset of $2^{1+k}$. The reader can easily check that such a triangulation $T$ always exists. The choice of the triangulation $T$ combined with Lemma 1 implies

Claim 3 Each simplex $\tau$ of the triangulation $T$ is covered by a suitable shift $x+2 \Xi_{s}^{k}$ of the homothetic copy $2 \Xi_{s}^{k}$ of the $\left(\begin{array}{l}k \\ s\end{array}\right)$-sandwich $\Xi_{s}^{k}$.

Let $\Delta$ be (the geometric realization of) a simplex in $\mathbb{R}^{k}$ with vertices $w_{0}, \ldots, w_{k}$ such that $w_{0}+\cdots+w_{k}=0$. The latter equality means that $\Delta$ is centered at the origin (which lies in the interior of $\Delta$ ). By $\Delta^{(0)}=\left\{w_{0}, \ldots, w_{k}\right\}$ we denote the set of vertices of the simplex $\Delta$.

Each point $y \in \Delta$ can be uniquely written as the convex combination $y=$ $\sum_{i=0}^{k} y_{i} w_{i}$ for some non-negative real numbers $y_{0}, \ldots, y_{k}$ with $\sum_{i=0}^{k} y_{i}=1$. The set

$$
\operatorname{supp}(y)=\left\{i \in\{0, \ldots, k\}: y_{i} \neq 0\right\}
$$

is called the support of $y$. It is clear that $\operatorname{supp}(y)$ is the smallest subset of $\Delta^{(0)}$ whose convex hull contains the point $y$.

Identifying each number $i \in\{0, \ldots, k\}$ with the vertex $w_{i}$ of $\Delta$, we can think of the coloring $\chi: \mathbb{Z}^{1+k} \rightarrow\{0, \ldots, k\}$ as a function $\chi: \mathbb{Z}^{1+k} \rightarrow \Delta^{(0)}=\left\{w_{0}, \ldots, w_{k}\right\}$.

Now extend the restriction $\chi \mid \partial K \cap(2 \mathbb{Z})^{1+k}$ of $\chi$ to a simplicial map $f: \partial K \rightarrow \Delta$ (which is affine on the convex hull of each simplex $\tau \in T$ ). The simpliciality of $f$ implies

Claim 4 For each simplex $\tau \in T$ and a point $x \in \operatorname{conv}(\tau)$

$$
\operatorname{supp}(f(x)) \subset \chi(\tau) \subset \chi\left(\lfloor x\rfloor+2 \cdot \mathbf{2}^{J_{x}}\right) .
$$


This claim has the following corollary.

Claim $5 f(\partial K) \subset \partial \Delta$.

Proof Given any point $x \in \partial K$, find a simplex $\tau \in T$ whose convex hull contains $x$. By the choice of the triangulation $T$ and Lemma 1, $\tau \subset-y+2 \Xi_{s}^{k}$ for some point $y \in \mathbb{Z}^{1+k}$. By Claim 2, $\chi(-y) \notin \chi(\tau)$ and thus

$$
f(x) \in \operatorname{conv}(f(\tau))=\operatorname{conv}(\chi(\tau)) \subset \operatorname{conv}\left(\Delta^{(0)} \backslash \chi(-y)\right) \subset \partial \Delta .
$$

Now consider the intersection $K_{0}=\{0\} \times[-2 n, 2 n]^{k}$ of the cube $K$ with the hyperplane $\{0\} \times \mathbb{R}^{k}$, which will be identified with the space $\mathbb{R}^{k}$, and let $\partial K_{0}=$ $\partial K \cap \mathbb{R}^{k}$ be the boundary of $K_{0}$.

For each subset $J \subset k+1=\{0, \ldots, k\}$ consider the map

$$
p_{J}: \mathbb{R}^{1+k} \rightarrow \mathbb{R}, \quad p_{J}:\left(x_{i}\right)_{i=0}^{k} \mapsto 1 \cdot \prod_{j \in J} x_{j} .
$$

Here we assume that $p_{\emptyset}(x)=1$. It follows that $\sum_{J \subset k+1} p_{J}(x)>0$ for all $x \in$ $[0,2]^{k+1}$.

We remind that for a point $x \in \mathbb{R}^{1+k}, J_{x}=\left\{i \in\{0, \ldots, k\}: x_{i} \notin 2 \mathbb{Z}\right\}$ and $\lfloor x\rfloor$ stands for the unique point in $(2 \mathbb{Z})^{1+k}$ such that $x \in\lfloor x\rfloor+\mathbf{2}^{J_{x}}$ where $\mathbf{2}^{J}=\left\{\left(x_{i}\right)_{i=0}^{k} \in\right.$ $\left.2^{k+1}: \forall i \notin J\left(x_{i}=0\right)\right\}$.

Now consider the map $\varphi: \partial K_{0} \rightarrow \Delta$ defined by the formula

$$
\varphi(x)=\frac{\sum_{J \subset k+1} p_{J}(x-\lfloor x\rfloor) \cdot \chi\left(\lfloor x\rfloor+\mathbf{e}_{J}\right)}{\sum_{J \subset k+1} p_{J}(x-\lfloor x\rfloor)} .
$$

It can be shown that the map $\varphi$ is well-defined and continuous.

Claim $6 \operatorname{supp}(\varphi(x))=\chi\left(\lfloor x\rfloor+2 \cdot 2^{J_{x}}\right) \subset \chi\left(\lfloor x\rfloor+2 \Xi_{s}^{k}\right)$ for all $x \in \partial K_{0}$.

Proof Let $x \in \partial K_{0}$ be any point. The definition of $\varphi$ implies that $\operatorname{supp}(\varphi(x))=$ $\chi\left(\lfloor x\rfloor+\mathbf{2}^{J_{x}}\right)$. The inclusion $x \in \partial K_{0}$ implies that the set $J_{x}=\{j \in\{0, \ldots, k\}$ : $\left.\operatorname{pr}_{j}(x) \notin 2 \mathbb{Z}\right\}$ has cardinality $\left|J_{x}\right|<k$ and thus $\mathbf{2}^{J_{x}} \subset\{0\} \times \mathbf{2}_{<k}^{k} \subset \boldsymbol{\Xi}_{s}^{k}$. Consequently, $\lfloor x\rfloor+2 \cdot \mathbf{2}^{J_{x}} \subset\lfloor x\rfloor+2 \Xi_{s}^{k}$ and $\chi\left(\lfloor x\rfloor+2 \cdot \mathbf{2}^{J_{x}}\right) \subset \chi\left(\lfloor x\rfloor+2 \Xi_{s}^{k}\right)$.

Claim $7 \varphi(x) \neq \varphi(-x)$ for all $x \in \partial K_{0}$.

Proof Observe that $J_{x}=J_{-x}$ and $\lfloor-x\rfloor=-\lfloor x\rfloor-2 \mathbf{e}_{J_{x}}$. By Claim 6,

$$
\chi(-\lfloor x\rfloor)=\chi\left(\lfloor-x\rfloor+2 \cdot \mathbf{e}_{-J_{x}}\right) \in \chi\left([-x]+2 \cdot 2^{J_{-x}}\right)=\operatorname{supp}(\varphi(-x)) .
$$

On the other hand, Claim 1 guarantees that

$$
\chi(-\lfloor x\rfloor) \not \supset \chi\left(\lfloor x\rfloor+2 \Xi_{s}^{k}\right) \supset \chi\left(\lfloor x\rfloor+2 \cdot 2^{J_{x}}\right)=\operatorname{supp}(\varphi(x)) .
$$

Consequently, $\operatorname{supp}(\varphi(-x)) \neq \operatorname{supp}(\varphi(x))$ and $\varphi(x) \neq \varphi(-x)$. 
Finally, consider the homotopy

$$
\left(f_{t}\right): \partial K_{0} \times[0,1] \rightarrow \Delta, \quad f_{t}: x \mapsto t \varphi(x)+(1-t) f(x),
$$

connecting the map $f=f_{0}$ with the map $\varphi=f_{1}$.

Claim $8 \operatorname{supp}\left(f_{t}(x)\right) \subset \chi\left(\lfloor x\rfloor+2 \cdot \mathbf{2}^{J_{x}}\right) \subset \partial \Delta$ for all $x \in \partial K_{0}$ and $t \in[0,1]$.

Proof The inclusion $\operatorname{supp}\left(f_{t}(x)\right) \subset \chi\left(\lfloor x\rfloor+2 \cdot \mathbf{2}^{J_{x}}\right)$ follows from Claims 4 and 6.

The inclusion $x \in \partial K_{0}$ implies that the set $J_{x}=\left\{j \in\{0, \ldots, k\}: \operatorname{pr}_{j}(x) \notin 2 \mathbb{Z}\right\}$ has cardinality $\left|J_{x}\right|<k$ and thus $\mathbf{2}^{J_{x}} \subset\{0\} \times \mathbf{2}_{<k}^{k} \subset \Xi_{s}^{k}$. By Claim 1, $\chi(-\lfloor x\rfloor) \notin$ $\chi\left(\lfloor x\rfloor+2 \Xi_{s}^{k}\right)$ and then

$$
\begin{aligned}
f_{t}(x) & \in \operatorname{conv}\left(\operatorname{supp}\left(f_{t}(x)\right) \subset \operatorname{conv}\left(\chi\left(\lfloor x\rfloor+2 \cdot 2^{J_{x}}\right)\right)\right. \\
& \subset \operatorname{conv}\left(\chi\left(\lfloor x\rfloor+2 \Xi_{s}^{k}\right)\right) \subset \operatorname{conv}\left(\Delta^{(0)} \backslash \chi(-\lfloor x\rfloor)\right) \subset \partial \Delta .
\end{aligned}
$$

Let $S^{k-1}=\left\{x \in \mathbb{R}^{k}:\|x\|=1\right\}$ be the unit sphere in $\mathbb{R}^{k}$ with respect to the Euclidean norm $\|\cdot\|$ and $r: \mathbb{R}^{k} \backslash\{0\} \rightarrow S^{k-1}, r: x \mapsto x /\|x\|$, be the radial retraction. Observe that its restriction $r \mid \partial \Delta$ to the boundary of the geometric simplex $\Delta$ is a homeomorphism.

By Claim 5, $f(\partial K) \subset \partial \Delta \subset \mathbb{R}^{k} \backslash\{0\}$, so we can consider the map $g_{0}: \partial K \rightarrow$ $S^{k-1}$ defined by $g_{0}(x) \mapsto r \circ f(x)=f(x) /\|f(x)\|$. By Claim 8 , the map $g_{0} \mid \partial K_{0}$ is homotopic to the map

$$
g_{1}: \partial K_{0} \rightarrow S^{k-1}, \quad g_{1}(x) \mapsto r \circ f_{1}(x)=r \circ \varphi(x) .
$$

It follows from Claim 7 that $g_{1}(x) \neq g_{1}(-x)$ for all $x \in \partial K_{0}$. This implies that the formula

$$
h_{t}(x)=\frac{g_{1}(x)-\operatorname{tg}_{1}(-x)}{\left\|g_{1}(x)-\operatorname{tg}_{1}(-x)\right\|}, \quad x \in \partial K_{0}, t \in[0,1],
$$

determines a well-defined homotopy $\left(h_{t}\right): \partial K_{0} \rightarrow S^{k-1}$ connecting the map $g_{1}$ with the map

$$
h_{1}(x)=\frac{g_{1}(x)-g_{1}(-x)}{\left\|g_{1}(x)-g_{1}(-x)\right\|},
$$

which is antipodal in the sense that $h_{1}(-x)=-h_{1}(x)$. By [13, Chap. 4, Sect. 7.10], each antipodal map between spheres of the same dimension is not homotopically trivial. Consequently, the antipodal map $h_{1}: \partial K_{0} \rightarrow S^{k-1}$ is not homotopically trivial. On the other hand, $h_{1}$ is homotopic to the map $h_{0}=g_{1}$, which is homotopic to $g_{0} \mid \partial K_{0}$ and the latter map is homotopically trivial since the boundary $\partial K_{0}$ of the cube $K_{0}$ is contractible in the boundary $\partial K$ of $K$. This contradiction completes the proof of Theorem 5 . 


\section{$4 T$-shaped sets in $\mathbb{R}^{n}$}

Theorem 5, proved in the preceding section, yields an upper bound for the numbers $c_{k}\left(\mathbb{Z}^{k}\right)$. A lower bound for the numbers $c_{k}^{B}\left(\mathbb{R}^{k}\right)$ will be obtained by the technique of $T$-shaped sets created in [1].

Let $\mathbb{R}_{+}=[0, \infty)$ be the closed half-line. For every $n \geq 0$ consider the subset $T_{0} \subset \mathbb{R}^{0}$ defined inductively:

$$
\begin{aligned}
& T_{0}=\emptyset \subset \mathbb{R}^{0}=\{0\}, \quad T_{1}=\{0\} \subset \mathbb{R}^{1}, \quad \text { and } \\
& T_{n}=\left(\mathbb{R}^{n-1} \times\{0\}\right) \cup\left(T_{n-1} \times \mathbb{R}_{+}\right) \subset \mathbb{R}^{n}
\end{aligned}
$$

for $n>1$.

Definition 4 A subset $C \subset \mathbb{R}^{n}$ is called $T$-shaped if $f(C) \subset \mathbb{R} \times T_{n-1}$ for some affine transformation $f: \mathbb{R}^{n} \rightarrow \mathbb{R} \times \mathbb{R}^{n-1}$. The smallest cardinality of a subset $A \subset$ $\mathbb{R}^{n}$, which is not $T$-shaped is denoted by $t\left(\mathbb{R}^{n}\right)$.

Let us describe the geometric structure of $T$-shaped sets.

We say that for $k \leq n$, hyperplanes $H_{1}, \ldots, H_{k}$ in $\mathbb{R}^{n}$ are in general position if they are pairwise distinct and their normal vectors are linearly independent. This happens if and only if there is an affine transformation $f: \mathbb{R}^{n} \rightarrow \mathbb{R}^{n}$ that maps the $i$ th hyperplane onto the hyperplane $\mathbb{R}^{i-1} \times\{0\} \times \mathbb{R}^{n-i}$ for all $i \in\{1, \ldots, k\}$.

We shall say that a hyperplane $H \subset \mathbb{R}^{n}$ does not separate a subset $S \subset \mathbb{R}^{n+1}$ if $S$ lies in one of two closed half-spaces bounded by the hyperplane $H$. Such a hyperplane $H$ will be called non-separating for $S$. A hyperplane $H$ is called a support hyperplane for $S$ if $H \cap S \neq \emptyset$ and $H$ does not separate $S$.

Proposition 2 Let $n \in \mathbb{N}$. A subset $S \subset \mathbb{R}^{n+1}$ is $T$-shaped if and only if

$$
S \subset H_{1} \cup \cdots \cup H_{n}
$$

for some hyperplanes $H_{1}, \ldots, H_{n}$ in general position such that each hyperplane $H_{i}$, $1 \leq i \leq n$, does not separate the set $S \backslash\left(H_{1} \cup \cdots \cup H_{i-1}\right)$.

Proof This proposition can be easily derived from the equality

$$
\mathbb{R} \times T_{n}=\bigcup_{i=0}^{n-1} \mathbb{R}^{n-i} \times\{0\} \times \mathbb{R}_{+}^{i}
$$

that can be easily proved by induction on $n$.

By Lemma 7 of [1], $T$-shaped subsets of Euclidean spaces $\mathbb{R}^{k}$ are $k$-centerpole for Borel colorings. Consequently, $t\left(\mathbb{R}^{n}\right) \leq c_{n}^{B}\left(\mathbb{R}^{n}\right)$. This gives us a lower bound for the numbers $c_{k}^{B}\left(\mathbb{R}^{n}\right)$ and $c_{k}\left(\mathbb{R}^{n}\right)$ :

Proposition $3 t\left(\mathbb{R}^{k}\right) \leq c_{k}^{B}\left(\mathbb{R}^{k}\right) \leq c_{k}^{B}\left(\mathbb{R}^{n}\right) \leq c_{k}\left(\mathbb{R}^{n}\right)$ for any finite $k \leq n$. 
In the following theorem we collect all the available information on the numbers $t\left(\mathbb{R}^{n}\right)$.

\section{Theorem 6}

1. $t\left(\mathbb{R}^{1}\right)=1$,

2. $t\left(\mathbb{R}^{2}\right)=3$,

3. $t\left(\mathbb{R}^{3}\right)=6$,

4. $t\left(\mathbb{R}^{4}\right)=12$,

5. $t\left(\mathbb{R}^{n}\right) \leq n^{2}-n+1$ for every $n \geq 1$,

6. $t\left(\mathbb{R}^{n}\right) \geq t\left(\mathbb{R}^{n-1}\right)+n+1$ for any $n \geq 4$,

7. $t\left(\mathbb{R}^{n}\right) \geq \frac{1}{2}\left(n^{2}+3 n-4\right)$ for any $n \geq 4$.

Proof

1. Since $T_{0}=\emptyset$, a subset of $\mathbb{R}^{1}$ is $T$-shaped if and only if it is empty. Consequently, $t\left(\mathbb{R}^{1}\right)=1$.

2. Since $T_{1}=\{0\} \subset \mathbb{R}^{1}$, a subset $C \subset \mathbb{R}^{2}$ is $T$-shaped if and only if $C$ lies in an affine line. Consequently, $t\left(\mathbb{R}^{2}\right)=3$.

3. By Theorem 5, the 6-element $\left(\begin{array}{l}2 \\ 0\end{array}\right)$-sandwich $\Xi_{0}^{2}$ is 3-centerpole in $\mathbb{R}^{3}$. Consequently, $c_{3}\left(\mathbb{R}^{3}\right) \leq 6$. By Proposition $3, t\left(\mathbb{R}^{3}\right) \leq c_{3}\left(\mathbb{R}^{3}\right) \leq 6$. To see that $t\left(\mathbb{R}^{3}\right) \geq 6$, we need to check that a subset $C \subset \mathbb{R}^{3}$ of cardinality $|C| \leq 5$ is $T$-shaped, which means that after a suitable affine transformation of $\mathbb{R}^{3}, C$ can be embedded into $\mathbb{R} \times T_{2}$. By the definition, $T_{2}=\mathbb{R} \times\{0\} \cup\{0\} \times \mathbb{R}_{+}$.

Consider the convex hull $\operatorname{conv}(C)$ of $C$ in $\mathbb{R}^{3}$. If $C$ lies in an affine plane $H$, then applying to $\mathbb{R}^{3}$ a suitable affine transformation, we can assume that $C \subset H=$ $\mathbb{R} \times \mathbb{R} \times\{0\} \subset \mathbb{R} \times T_{2}$. If $C$ does not lie in a plane, then the convex polyhedron $\operatorname{conv}(C)$ has a supporting plane $H_{1}$ such that $\left|H_{1} \cap C\right| \geq 3$. So, $C \backslash H_{1}$ lies in one of the closed half-spaces with respect to the plane $H_{1}$. Denote this subspace by $H_{1}^{+}$. The set $C \backslash H_{1}$ has cardinality $\left|C \backslash H_{1}\right| \leq 2$ and hence it lies in an affine plane $H_{2} \subset \mathbb{R}^{3}$ that meets $H_{1}$. Find an affine transformation $f: \mathbb{R}^{3} \rightarrow \mathbb{R}^{3}$ such that $f\left(H_{1}\right)=\mathbb{R} \times \mathbb{R} \times\{0\}, f\left(H_{1}^{+}\right)=\mathbb{R} \times \mathbb{R} \times \mathbb{R}_{+}$and $f\left(H_{2}\right)=\{\mathbb{R}\} \times\{0\} \times\{\mathbb{R}\}$. Then

$$
f(C) \subset \mathbb{R} \times \mathbb{R} \times\{0\} \cup \mathbb{R} \times\{0\} \times \mathbb{R}_{+}=\mathbb{R} \times T_{2}
$$

and hence $C$ is $T$-shaped.

4. By Theorem 5 , the $\left(\begin{array}{l}3 \\ 1\end{array}\right)$-sandwich $\Xi_{1}^{3}$ is 4-centerpole in $\mathbb{Z}^{4}$. Consequently,

$$
t\left(\mathbb{R}^{4}\right) \leq c_{4}\left(\mathbb{R}^{4}\right) \leq c_{4}\left(\mathbb{Z}^{4}\right) \leq\left|\Xi_{1}^{3}\right|=2^{4}-1-\left(\begin{array}{l}
3 \\
1
\end{array}\right)=12 .
$$

The reverse inequality $t\left(\mathbb{R}^{4}\right) \geq 12$ will be proved in Lemma 2 below.

5. Let $C \subset \mathbb{R}^{n}$ be a set consisting of $n^{2}-n+1=n(n-1)+1$ points in general position. This means that no $(n+1)$-element subset of $C$ lies in a hyperplane. Then $C$ cannot be covered by less than $n$ hyperplanes and consequently $C$ is not $T$-shaped (because the set $\mathbb{R} \times T_{n-1}$ lies in the union of $(n-1)$ hyperplanes). Then $t\left(\mathbb{R}^{n}\right) \leq|C|=n^{2}-n+1$. 
6. First we prove the inequality

$$
t\left(\mathbb{R}^{n}\right) \geq \min \left\{2 t\left(\mathbb{R}^{n-1}\right), t\left(\mathbb{R}^{n-1}\right)+n+1\right\}
$$

for every $n \geq 2$. Take any subset $C \subset \mathbb{R}^{n}$ of cardinality $|C|<\min \left\{2 t\left(\mathbb{R}^{n-1}\right)\right.$, $\left.t\left(\mathbb{R}^{n-1}\right)+n+1\right\}$. We need to show that $C$ is $T$-shaped.

Consider the convex hull $\operatorname{conv}(C)$ of $C$ in $\mathbb{R}^{n}$. If $\operatorname{conv}(C)$ lies in some hyperplane, then $C$ is $T$-shaped by the definition. So, we assume that $\operatorname{conv}(C)$ does not lie in a hyperplane and then $\operatorname{conv}(C)$ is a compact convex body in $\mathbb{R}^{n}$. Let $H$ be a supporting hyperplane of $\operatorname{conv}(C)$ having maximal possible cardinality of the intersection $C \cap H$. It is clear that $|C \cap H| \geq n$.

Now two cases are possible:

(a) The set $C \backslash H$ lies in a hyperplane $H_{1}$, parallel to $H$. Then $H_{1}$ is a supporting hyperplane of $\operatorname{conv}(C)$ and then $\left|C \cap H_{1}\right| \leq|C \cap H|$ by the choice of $H$. Now we see that $\left|C \cap H_{1}\right| \leq \frac{1}{2}|C|<t\left(\mathbb{R}^{n-1}\right)$.

Applying to $\mathbb{R}^{n}=\mathbb{R}^{n-1} \times \mathbb{R}$ a suitable affine transformation, we can assume that $H=\mathbb{R}^{n-1} \times\{0\}$ and $C \backslash H \subset \mathbb{R}^{n-1} \times \mathbb{R}_{+}$. Let pr: $\mathbb{R}^{n} \rightarrow \mathbb{R}^{n-1}$ be the coordinate projection. Since $\left|\operatorname{pr}_{n}(C \backslash H)\right|<t\left(\mathbb{R}^{n-1}\right)$, the set $C^{\prime}=\operatorname{pr}_{n}(C \backslash H)$ is $T$ shaped. This means that there is an affine transformation $f: \mathbb{R}^{n-1} \rightarrow \mathbb{R}^{n-1}$ such that $f\left(C^{\prime}\right) \subset \mathbb{R} \times T_{n-2}$. This affine transformation $f$ induces the affine transformation

$$
\Phi: \mathbb{R}^{n-1} \times \mathbb{R} \rightarrow \mathbb{R}^{n-1} \times \mathbb{R}, \quad \Phi(x, y)=(f(x), y)
$$

such that

$$
\begin{aligned}
\Phi(C) & =\Phi(C \cap H) \cup \Phi(C \backslash H) \subset\left(\mathbb{R} \times \mathbb{R}^{n-2} \times\{0\}\right) \cup\left(\mathbb{R} \times T_{n-2} \times \mathbb{R}_{+}\right) \\
& =\mathbb{R} \times T_{n-1} .
\end{aligned}
$$

The affine transformation $\Phi$ witnesses that the set $C$ is $T$-shaped.

(b) The set $C \backslash H$ does not lie in a hyperplane parallel to $H$. Then $C \backslash H$ contains two distinct points $x, y$ such that the vector $\overrightarrow{x y}$ is not parallel to $H$. Applying to $\mathbb{R}^{n}=\mathbb{R}^{n-1} \times \mathbb{R}$ a suitable affine transformation, we can assume that $H=$ $\mathbb{R}^{n-1} \times\{0\}, C \backslash H \subset \mathbb{R}^{n-1} \times \mathbb{R}_{+}$, and under the projection pr : $\mathbb{R}^{n-1} \times \mathbb{R} \rightarrow \mathbb{R}^{n-1}$ the images of the points $x$ and $y$ coincide. Then the projection $C^{\prime}=\operatorname{pr}(C \backslash H)$ has cardinality $\left|C^{\prime}\right| \leq|C \backslash H|-1<|C|-|C \cap H|-1<t\left(\mathbb{R}^{n-1}\right)+n+1-n-1=$ $t\left(\mathbb{R}^{n-1}\right)$. Continuing as in the preceding case, we can find an affine transformation $\Phi$, witnessing that $C$ is a $T$-shaped set in $\mathbb{R}^{n}$.

This proves the inequality (1). By analogy we can prove that $t\left(\mathbb{R}^{n}\right) \geq$ $t\left(\mathbb{R}^{n-1}\right)+n$. Since $t\left(\mathbb{R}^{1}\right)=1$, by induction we can show that $t\left(\mathbb{R}^{n}\right) \geq \frac{1}{2} n(n+1)$. In particular, $t\left(\mathbb{R}^{n-1}\right) \geq \frac{1}{2} n(n-1) \geq n+1$ for all $n \geq 4$. In this case

$$
t\left(\mathbb{R}^{n}\right) \geq \min \left\{2 t\left(\mathbb{R}^{n-1}\right), t\left(\mathbb{R}^{n-1}\right)+n+1\right\}=t\left(\mathbb{R}^{n-1}\right)+n+1 .
$$

7. The lower bound $t\left(\mathbb{R}^{n}\right) \geq \frac{1}{2}\left(n^{2}+3 n-4\right), n \geq 4$, will be proved by induction. For $n=4$ it is true according to the statement (4). Assuming that it is true for some 
$n>4$ and applying the lower bound (6), we get

$$
\begin{aligned}
t\left(\mathbb{R}^{n+1}\right) & \geq t\left(\mathbb{R}^{n}\right)+(n+1)+1 \geq \frac{1}{2}\left(n^{2}+3 n-4\right)+n+2 \\
& =\frac{1}{2}\left((n+1)^{2}+3(n+1)-4\right) .
\end{aligned}
$$

To finish the proof of Theorem 6, it remains to prove the promised:

Lemma 2 Each subset $C \subset \mathbb{R}^{4}$ of cardinality $|C|<12$ is $T$-shaped.

Proof Assume that some subset $C \subset \mathbb{R}^{4}$ of cardinality $|C|<12$ is not $T$-shaped. Without loss of generality, $|C|=11$.

We recall that a hyperplane $H \subset \mathbb{R}^{4}$ is called a support hyperplane for $C$ if $C \cap$ $H \neq \emptyset$ and $H$ does not separate $C$ (which means that $C$ lies in a closed half-space $H^{+}$bounded by the hyperplane).

Claim 9 Each support hyperplane $H \subset \mathbb{R}^{4}$ for $C$ has at most five common points with $C$.

Proof Assume that $H$ is a support hyperplane for $C$ with $|H \cap C|>5$. After a suitable affine transformation of $\mathbb{R}^{4}$, we can assume that $H=\mathbb{R}^{3} \times\{0\}$ and $C \subset \mathbb{R}^{3} \times \mathbb{R}_{+}$. Let pr: $\mathbb{R}^{4} \rightarrow \mathbb{R}^{3}$ be the coordinate projection. Since $|C \backslash H|=|C|-|C \cap H|<$ $11-5=6$ and $t\left(\mathbb{R}^{3}\right)=6$ (by Theorem $\left.6(3)\right), \operatorname{pr}(C \backslash H)$ is $T$-shaped in $H$ and so $C$ is $T$-shaped $\mathbb{R}^{4}$.

Claim 10 For any two parallel hyperplanes $H_{1}$ and $H_{2}$ in $\mathbb{R}^{4}$ the set $C \backslash\left(H_{1} \cup H_{2}\right)$ is non-empty.

Proof Otherwise one of these hyperplanes contains more than six points, which contradicts Claim 9.

Claim 11 Each support hyperplane $H$ for the set $C$ has less than five common points with $C$.

Proof Previous claim guarantees the existence of two distinct points $a, b \in C$ that lie in an affine line $L$ that meets $H$. After a suitable affine transformation of $\mathbb{R}^{4}$, we can assume that $H=\mathbb{R}^{3} \times\{0\}, C \subset \mathbb{R}^{3} \times \mathbb{R}_{+}$, and $L=\{0\}^{3} \times \mathbb{R}$. Let pr: $\mathbb{R}^{4} \rightarrow \mathbb{R}^{3}$ be the coordinate projection. Assuming that $|H \cap C| \geq 5$ and taking into account that $\operatorname{pr}(a)=\operatorname{pr}(b)$, we conclude that

$$
|\operatorname{pr}(C \backslash H)| \leq|C \backslash H|-1=|C|-|C \cap H|-1 \leq 5<6=t\left(\mathbb{R}^{3}\right) .
$$

It follows that $\operatorname{pr}(C \backslash H)$ is $T$-shaped in $\mathbb{R}^{3}$ and then $C$ is $T$-shaped in $\mathbb{R}^{4}$.

The characterization of $T$-shaped sets given in Proposition 2 implies: 
Claim 12 If $H_{1}$ is a support hyperplane for $C, H_{2}$ is a support hyperplane for $C \backslash H_{1}$ and $H_{1}, H_{2}$ are not parallel, then $\left|C \backslash\left(H_{1} \cup H_{2}\right)\right| \geq 3$ and if $\left|C \backslash\left(H_{1} \cup H_{2}\right)\right|=3$, then the set $C \backslash\left(H_{1} \cup H_{2}\right)$ does not lie in a line but lies in a plane, parallel to $H_{1} \cap H_{2}$.

Claim 13 If $H_{1}$ and $P_{2}$ are parallel support hyperplanes for $C$ and $\left|H_{1} \cap C\right|=4$, then $\left|P_{2} \cap C\right|=1$.

Proof By Claim 11, $C \backslash H_{1}$ does not lie in a hyperplane. Now consider four cases.

(1) $\left|P_{2} \cap C\right|>4$. In this case $C$ is $T$-shaped by Claim 11 .

(2) $\left|P_{2} \cap C\right|=4$. We claim that the set $P_{2} \cap C$ does not lie in a plane $P$. Otherwise $P$ can be enlarged to a support hyperplane that contains $\geq 5$ points of $C$, which is forbidden by Claim 11. Therefore, the convex hull of $P_{2} \cap C$ is a convex body in $P_{2}$ and we can find a support hyperplane $H_{2}$ for $C \backslash H_{1}$ that meets $H_{1}$, has at least four common points with $C \backslash H_{1}$ and exactly three common points with the set $C \cap P_{2}$. In this case the unique point $c_{2}$ of the set $C \cap P_{2} \backslash H_{2}$ lies in $C \backslash\left(H_{1} \cup H_{2}\right)$. By Proposition 2, the set $C \backslash\left(H_{1} \cup H_{2}\right)$ contains exactly three points that lie in a plane parallel to $H_{1} \cap H_{2}$. Since this set contains the point $c_{2} \in C \cap P_{2}$, we conclude that $C \backslash\left(H_{1} \cup H_{2}\right) \subset P_{2}$ and hence $\left|C \cap P_{2}\right|=6$, which is a contradiction.

(3) $\left|P_{2} \cap C\right|=3$. Let $P l$ be a plane which contains $P_{2} \cap C$ and lies in the hyperplane $P_{2}$. We claim that the set $C \backslash\left(H_{1} \cup P l\right)$ lies in a plane $P l_{1}$ that is parallel to $P l$. Let $S$ be the set of all points $x \in C \backslash\left(H_{1} \cup P l\right)$ that belong to a support hyperplane $H_{x}$ to $C \backslash H_{1}$ that has at least four common points with $C \backslash H_{1}$ and contains the plane $P l$. Claim 12 guarantees that the set $C \backslash\left(H_{1} \cup H_{x}\right)$ contains exactly three elements and lies in a plane that is parallel to the intersection $H_{1} \cap H_{x}$ (which is parallel to $P l$ ). Since the set $C \backslash H_{1}$ does not lie in a hyperplane, the set $S$ contains more that one point, which implies that the set $C \backslash\left(H_{1} \cup P l\right)=$ $\bigcup_{x \in S} C \backslash\left(H_{1} \cup H_{x}\right)$ lies in a plane $P l_{1}$ that is parallel to the plane $P l$. Let $H_{2}$ be the hyperplane that contains the parallel planes $P l$ and $P l_{1}$. Since $H_{2}$ meets $H_{1}$, we see that $C \subset H_{1} \cup H_{2}$ is $T$-shaped by Proposition 2 and this is a contradiction.

(4) $\left|P_{2} \cap C\right|=2$. Since $C \backslash H_{1}$ does not lie in a hyperplane, there is a support hyperplane $H_{2}$ to $C \backslash H_{1}$ such that $\left|H_{2} \cap\left(C \backslash H_{1}\right)\right| \geq 4$ and $\left|H_{2} \cap P_{2} \cap C\right|=1$. It follows that the hyperplane $H_{2}$ does not coincide with $P_{2}$ and hence meets the hyperplane $H_{1}$. By Claim 12, the complement $C \backslash\left(H_{1} \cup H_{2}\right)$ contains exactly three points that lie in a plane, parallel to $H_{1} \cap H_{2}$. Since $C \backslash\left(H_{1} \cup H_{2}\right)$ meets the hyperplane $P_{2}$ we conclude that $C \backslash\left(H_{1} \cup H_{2}\right) \subset P_{2}$ and $\left|C \cap P_{2}\right| \geq 4$, which is a contradiction.

Claim 14 If $P_{1}$ and $P_{2}$ are parallel support hyperplanes for $C$ and $\left|P_{1} \cap C\right|=4$, then the set $C \backslash\left(P_{1} \cup P_{2}\right)$ lies in a hyperplane $P_{3}$ that is parallel to $P_{1}$ and $P_{2}$.

Proof By Claim 13, $\left|P_{2} \cap C\right|=1$ and hence $\left|C \backslash\left(P_{1} \cup P_{2}\right)\right|=6$. Let $x$ be the unique point of $P_{2} \cap C$. Take any support hyperplane $H \ni x$ for the set $C \backslash P_{1}$ such that $|H \cap C| \geq 4$. Since $H$ meets $P_{1}$, Proposition 2 guarantees that the set $C^{\prime}=C \backslash$ $\left(P_{1} \cup H\right)$ contains exactly three points that lie in a plane parallel to the intersection 
$P_{1} \cap H$ and hence parallel to $P_{1}$. The hyperplane $H^{\prime}$ containing the set $C^{\prime} \cup\{x\}$ is a support hyperplane for the set $C \backslash P_{1}$. Applying Proposition 2, we conclude that the set $C^{\prime \prime}=C \backslash\left(P_{1} \cup H^{\prime}\right)=C \cap H \backslash P_{2}$ contains exactly three points lying in a plane parallel to $P_{1} \cap H^{\prime}$. Thus $C \backslash\left(P_{1} \cup P_{2}\right)$ lies in two planes parallel to $P_{1}$ and hence it lies in a hyperplane $P_{3}$. Proposition 2 implies that the hyperplane $P_{3}$ is parallel to $P_{1}$.

By an octahedron in a linear space $L$ we understand a set of the form

$$
c+\left\{\mathbf{e}_{i},-\mathbf{e}_{i}: 1 \leq i \leq 3\right\}
$$

where $\mathbf{e}_{1}, \mathbf{e}_{2}, \mathbf{e}_{3}$ are linearly independent vectors in $L$ and $c \in L$ is the center of the octahedron. Up to an affine equivalence an octahedron is a unique 6-element set $X$ with 3-dimensional affine hull $A$ such that for each support plane $P \subset A$ of $X$ with $|P \cap X| \geq 3$ the set $X \backslash P$ contains three points and lies in a plane $P^{\prime}$, parallel to $P$.

Claim 15 If $P_{1}$ and $P_{2}$ are parallel support hyperplanes for $X$ and $\left|P_{1} \cap C\right|=4$, then the set $C \backslash\left(P_{1} \cup P_{2}\right)$ is an octahedron that lies in a hyperplane $P_{3}$, parallel to $P_{1}$.

Proof By the preceding claim, the set $K=C \backslash\left(P_{1} \cup P_{2}\right)$ lies in a hyperplane $P_{3}$, parallel to $P_{1}$. Let us show that $K$ does not lie in a plane. In the opposite case, we could find a hyperplane $H_{2}$ that contains the set $K$ and meets the hyperplane $P_{1}$. Then for each hyperplane $H_{3}$ that contains the unique point $C \cap P_{2}$ and has onedimensional intersection with $P_{1} \cap H_{2}$, we get $C \subset P_{1} \cup H_{2} \cup H_{3}$ witnessing that $C$ is $T$-shaped.

Thus the affine hull of $K$ is 3-dimensional. To see that $K$ is an octahedron, it suffices to check that for each support plane $P \subset P_{3}$ of $K$ with $|P \cap K| \geq 3$ the set $K \backslash P$ contains exactly three points and lies in a plane parallel to $P$.

Let $x$ be the unique point of the set $C \cap P_{2}$ and $H_{2}$ be the hyperplane containing the plane $P$ and passing through $x$. It follows that $H_{2}$ is a support hyperplane for the set $C \backslash P_{1}$. By Claim 12, the set $C \backslash\left(P_{1} \cup H_{2}\right)=K \backslash P$ contains exactly three elements and lies in a plane $P^{\prime}$ parallel to the intersection $H_{1} \cap H_{2}$.

Now let $H_{2}^{\prime}$ be the hyperplane that contains the support plane $P^{\prime}$ and passes through the point $x$. Since $P^{\prime}$ is a support plane for $K$ in the hyperplane $P_{3}, H_{3}$ is a support hyperplane for $K \cup\{x\}=C \backslash P_{1}$ in $\mathbb{R}^{4}$. Since $H_{3}^{\prime}$ intersects $P_{1}$, Claim 12 guarantees that the set $C \backslash\left(P_{1} \cup H_{2}^{\prime}\right)=K \backslash P^{\prime}$ contains exactly three points and the plane $P$ containing these three points is parallel to $P_{1} \cap H_{2}^{\prime}$ which is parallel to the plane $P^{\prime}$.

After this preparatory work we are ready to finish the proof of Lemma 2 . As $C$ is not $T$-shaped, it does not lie in a hyperplane. So, we can find a support hyperplane $P_{1}$ for $C$ such that $\left|P_{1} \cap C\right| \geq 4$. Let $P_{2}$ be a support hyperplane for $C$, which is parallel to $P_{1}$. By Claim 13, $\left|P_{1} \cap C\right|=4$ and $\left|P_{2} \cap C\right|=1$. Let $p_{2}$ be the unique point of the set $P_{2} \cap C$. By Claim 15, $C \backslash\left(P_{1} \cup P_{2}\right)$ is an octahedron that lies in a hyperplane $P_{3}$, parallel to the hyperplanes $P_{1}$ and $P_{2}$. Let $c$ be the center of this octahedron and $2 c-p_{2}$ be the point, symmetric to $p_{2}$ with respect to $c$. 
Fix any 3-element subset $F$ of $P_{1} \cap C$ such that $2 c-p_{2} \in F$ if $2 c-p_{2} \in C \cap P_{1}$. Next, find a hyperplane $H_{1}$ for $C$ that contains $F$ and meets $C \backslash H_{1}$ at some point $a$. If $a=p_{2}$, then the set $C \subset H_{1} \cup P_{3} \cup\left(C \cap P_{1} \backslash F\right)$ is $T$-shaped by Proposition 2 .

Consequently, $a$ is a point of the octahedron $C \cap P_{3}$ with center $c$. Let $H_{2}$ be a support hyperplane for $C$ that is parallel to the hyperplane $H_{1}$. By Claims 13 and 15 , $\left|C \cap H_{1}\right|=4,\left|C \cap H_{2}\right|=1$ and $C \backslash\left(H_{1} \cup H_{2}\right)$ is an octahedron that lies in a hyperplane $H_{3}$, parallel to $H_{1}$ and $H_{2}$. If $H_{3}$ does not meet the octahedron $C \cap P_{3}$, then $\left(C \cap P_{3}\right) \cap\left(C \cap H_{3}\right)=\left(C \cap P_{3}\right) \backslash H_{1}=C \cap P_{3} \backslash\{a\}$. In this case the octahedra $C \cap P_{3}$ and $C \cap H_{3}$ have five common points and hence lie in the same hyperplane $P_{3}=H_{3}$, which is not possible. So, the support hyperplane $H_{3}$ meets the octahedron $C \cap P_{3}$ at a single point and this point is $2 c-a$. In this case the octahedra $C \cap P_{3}$ and $C \cap H_{3}$ have four common points which belong to the set $C \cap P_{3} \backslash\{a, 2 c-a\}$ and lie in the 2-dimensional plane $P_{3} \cap H_{3}$. This implies that the octahedra $C \cap P_{3}$ and $C \cap H_{3}$ have the common center $c$. Since $p_{2} \in C \cap H_{3}$, the point $2 c-p_{2}$ belongs to the octahedron $C \cap H_{3} \subset C$. It follows from $p_{2} \in P_{2}$ and $c \in P_{3}$ that $2 c-p_{2} \in C \backslash\left(P_{2} \cup P_{3}\right)=C \cap P_{1}$ and hence $2 c-p_{2} \subset F \subset H_{1}$ by the choice of the set $F$. On the other hand, $2 c-p_{2}$ belongs to the hyperplane $H_{3}$, which is disjoint with $H_{1}$ and this is a desired contradiction.

\section{Enlarging non-centerpole sets}

In this section we prove several lemmas on enlarging non-centerpole subsets. Namely, we show that under certain conditions, a non- $k$-centerpole subset $C$ of a topological group $X$ (possibly enlarged by one or two points) remains not $k$-centerpole in the direct sum $X \oplus \mathbb{R}$. The group $X \oplus \mathbb{R}$ can be identified with the direct product $X \times \mathbb{R}$ so that $X$ is identified with the subgroup $X \times\{0\} \subset X \times \mathbb{R}$, while the real line $\mathbb{R}$ is identified with the subgroup $\{e\} \times \mathbb{R} \subset X \times \mathbb{R}$ where $e$ is the neutral element of the group $X$.

Lemma 3 If for $k \geq 2$ a subset $C \subset X$ of a topological group $X$ is not $k$-centerpole (for Borel colorings), then set $C$ is not $k$-centerpole in $X \oplus \mathbb{R}$.

Proof Since the set $C \subset X$ is not $k$-centerpole (for Borel colorings), there exists a (Borel) coloring $\chi: X \rightarrow k$ such that $X$ contains no monochromatic unbounded subset, which is symmetric with respect to a point $c \in C$. Extend $\chi$ to a (Borel) coloring $\tilde{\chi}: X \times \mathbb{R} \rightarrow k$ letting

$$
\tilde{\chi}(x, t)= \begin{cases}\chi(x) & \text { if } t=0, \\ 0 & \text { if } t<0, \\ 1 & \text { if } t>0 .\end{cases}
$$

This coloring witnesses that $C$ is not $k$-centerpole in $X \oplus \mathbb{R}$ (for Borel colorings).

Lemma 4 If for $k \geq 3$ a subset $C \subset X$ of a topological group $X$ with $c_{2}^{B}(X) \geq 2$ is not $k$-centerpole (for Borel colorings), then for each $x \in X \times(0, \infty)$ the set $C \cup\{x\}$ is not $k$-centerpole for (Borel) colorings of the topological group $X \oplus \mathbb{R}$. 
Proof Without loss of generality we may assume that $x=(e, 1)$ where $e$ is the neutral element of topological group $X$. Fix a (Borel) coloring $\chi: X \rightarrow k$ witnessing that the subset $C \subset X$ is not $k$-centerpole (for Borel colorings).

This coloring induces a (Borel) 2-coloring $\chi_{2}: X \rightarrow 2$ defined by

$$
\chi_{2}(x)=\min \left(\{0,1\} \backslash \chi\left(x^{-1}\right)\right) \text { for } x \in X .
$$

Since $c_{2}^{B}(X) \geq 2$, there exists a Borel coloring $\chi_{1}: X \rightarrow 2$ witnessing that the singleton $\{e\}$ is not 2-centerpole for Borel colorings of $X$.

It is easy to see that the (Borel) coloring $\tilde{\chi}: X \times \mathbb{R} \rightarrow k$ defined by

$$
\tilde{\chi}(x, t)= \begin{cases}\chi(x), & \text { if } t=0, \\ \chi_{1}(x), & \text { if } t=1, \\ \chi_{2}(x), & \text { if } t=2, \\ 0, & \text { if } 1<t \neq 2, \\ 1, & \text { if } 0<t<1, \\ 2 & \text { if } t<0\end{cases}
$$

witnesses that the set $C \cup\{(e, 1)\}$ fails to be $k$-centerpole for (Borel) colorings of the topological group $X \oplus \mathbb{R}$.

Lemma $5 c_{3}^{B}\left(\mathbb{R}^{m}\right) \geq 6$ for all $m \geq 3$.

Proof By Theorem 6(3) and Proposition 3, $c_{3}^{B}\left(\mathbb{R}^{3}\right) \geq t\left(\mathbb{R}^{3}\right)=6$.

Next, we check that $c_{3}^{B}\left(\mathbb{R}^{4}\right) \geq 6$. Assuming that $c_{3}^{B}\left(\mathbb{R}^{4}\right)<6$ find a subset $C \subset \mathbb{R}^{4}$ of cardinality $|C| \leq 5$, which is 3-centerpole for Borel colorings of $\mathbb{R}^{4}$.

Since $|C| \leq 5$, there is a 3-dimensional hyperplane $H_{3} \subset \mathbb{R}^{4}$ such that $\left|C \backslash H_{3}\right| \leq 1$. Since $\left|C \cap H_{3}\right| \leq|C|<6=c_{3}^{B}\left(\mathbb{R}^{3}\right)$, the set $C \cap H_{3}$ is not 3-centerpole for Borel colorings of $H_{3}$. By (the proof of) Proposition 4.1 of [3], $c_{2}^{B}\left(\mathbb{R}^{3}\right)=3 \geq 2$. By Lemma 4, the set $C$ is not 3-centerpole for Borel colorings of $H_{3} \oplus \mathbb{R}$ (which can be identified with $\mathbb{R}^{4}$ ).

Now assume that the inequality $c_{3}^{B}\left(\mathbb{R}^{m-1}\right) \geq 6$ has been proved for some $m \geq$ 4. Assuming that $c_{3}^{B}\left(\mathbb{R}^{m}\right) \leq 5$ find a subset $C \subset \mathbb{R}^{m}$ of cardinality $|C| \leq 5$ which is 3-centerpole for Borel colorings of $\mathbb{R}^{m}$. This set lies in an $(m-1)$-dimensional hyperplane and according to Lemma 3, is 3-centerpole for Borel colorings of $\mathbb{R}^{m-1}$. Then $c_{3}^{B}\left(\mathbb{R}^{m-1}\right) \leq|C| \leq 5$, which contradicts the inductive assumption.

Lemma 6 If for $k \geq 4$ a subset $C \subset X$ of a topological group $X$ with $c_{2}^{B}(X) \geq 3$ is not $k$-centerpole (for Borel colorings), then for any 2-element set $A \subset X \times(0, \infty)$ the set $C \cup A$ is not $k$-centerpole for (Borel) colorings of the topological group $X \oplus \mathbb{R}$.

Proof Let $(a, v)$ and $(b, w)$ be the points of the 2-element set $A \subset X \times(0, \infty)$. We can assume that $v \leq w$. Let $\chi_{0}: X \rightarrow k$ be a (Borel) coloring witnessing that the set $C$ is not $k$-centerpole for (Borel) colorings of the group $X$. 
Consider the Borel 4-coloring $\psi: \mathbb{R} \rightarrow 4$ of the real line defined by

$$
\psi(t)= \begin{cases}3 & \text { if } t \leq 0 \\ 0 & \text { if } 0<t \leq v \\ 1 & \text { if } v<t \leq w \\ 2 & \text { if } w<t\end{cases}
$$

and observe that for each $c \in\{0, v, w\}$ and $t \in \mathbb{R} \backslash\{c\}$ we get $\psi(t) \neq \psi(2 c-t)$.

We consider two cases.

(1) $v=w$. In this case we can assume that $v=w=1$. Since $c_{2}^{B}(X) \geq 3$, there exists a Borel coloring $\chi_{1}: X \rightarrow 2$ witnessing that the 2-element set $\{a, b\} \subset X$ is not 2centerpole for Borel colorings of $X$. The (Borel) coloring $\chi_{0}$ induces the (Borel) coloring $\chi_{2}: X \rightarrow 3$ defined by the formula

$$
\chi_{2}(x)=\min \left(\{0,1,2\} \backslash\left\{\chi_{0}\left(a x^{-1} a\right), \chi_{0}\left(b x^{-1} b\right)\right\}\right) .
$$

Now we see that the (Borel) coloring $\tilde{\chi}: X \times \mathbb{R} \rightarrow k$ defined by

$$
\tilde{\chi}(x, t)= \begin{cases}\chi_{t}(x), & \text { if } t \in\{0,1,2\}, \\ \psi(t), & \text { otherwise }\end{cases}
$$

witnesses that the set $C \cup A$ is not $k$-centerpole for (Borel) colorings of the topological group $X \oplus \mathbb{R}$.

(2) The second case occurs when $v \neq w$. Without loss of generality, $v<w$ and $w-$ $v=1$. This case has three subcases.

(2a) $v=1$ and $w=2$. In this case we can assume that $b=e$ is the neutral element of the group $X$.

Since $c_{2}^{B}(X) \geq 3$, there is a Borel 2-coloring $\chi_{1}: X \rightarrow 2$ witnessing that the singleton $\{a\}$ is not 2-centerpole in $X$. By the same reason, there is a Borel 2-coloring $\phi: X \rightarrow 2$ witnessing that the singleton $\{b\}=\{e\}$ is not 2-centerpole for Borel colorings of $X$. Using the colorings $\phi$ and $\chi_{0}$ one can define a (Borel) 3-coloring $\chi_{2}: X \rightarrow 3$ such that $\chi_{2}(x) \neq \chi_{0}\left(a x^{-1} a\right)$ for all $x \in X$ and $\chi_{2}(x) \neq \chi_{2}\left(x^{-1}\right)$ if and only if $\phi(x) \neq \phi\left(x^{-1}\right)$.

Such a coloring $\chi_{2}: X \rightarrow 3$ can be defined by the formula

$$
\chi_{2}(x)=\left\{\begin{array}{l}
\min \left(3 \backslash\left\{\chi_{0}(a x a), \chi_{0}\left(a x^{-1} a\right)\right\}\right), \quad \text { if } \phi(x)=\phi\left(x^{-1}\right) \\
\phi(x), \\
\quad \text { if } \chi_{0}\left(a x^{-1} a\right) \neq \phi(x) \neq \phi\left(x^{-1}\right) \neq \chi_{0}(\text { axa }) \\
\min \left(3 \backslash\left\{\phi\left(x^{-1}\right), \chi_{0}\left(a x^{-1} a\right)\right\}\right), \\
\quad \text { if } \chi_{0}\left(a x^{-1} a\right)=\phi(x) \neq \phi\left(x^{-1}\right) \neq \chi_{0}(\text { axa }) \\
\phi(x) \\
\quad \text { if } \chi_{0}\left(a x^{-1} a\right) \neq \phi(x) \neq \phi\left(x^{-1}\right)=\chi_{0}(\text { axa } a) \\
\phi\left(x^{-1}\right) \\
\quad \text { if } \chi_{0}\left(a x^{-1} a\right)=\phi(x) \neq \phi\left(x^{-1}\right)=\chi_{0}(\text { axa } a) .
\end{array}\right.
$$


Let $\chi_{3}: X \rightarrow 2$ be the Borel 2-coloring defined by $\chi_{3}(x)=1-\chi_{1}\left(x^{-1}\right)$ for $x \in X$. It is clear that $\chi_{3}\left(x^{-1}\right) \neq \chi_{1}(x)$ for all $x \in X$. Finally, consider the Borel 2-coloring $\chi_{4}: X \rightarrow 2$ defined by

$$
\chi_{4}(x)=\min \left(\{0,1\} \backslash\left\{\chi_{0}\left(x^{-1}\right)\right\}\right) \quad \text { for } x \in X .
$$

The (Borel) colorings $\psi, \chi_{0}, \chi_{1}, \chi_{2}, \chi_{3}, \chi_{4}$ compose a (Borel) $k$-coloring $\tilde{\chi}: X \times \mathbb{R} \rightarrow k$,

$$
\tilde{\chi}(x, t)= \begin{cases}\chi_{t}(x), & \text { if } t \in\{0,1,2,3,4\}, \\ \psi(t), & \text { otherwise, }\end{cases}
$$

witnessing that the set $C \cup A$ is not $k$-centerpole for (Borel) colorings of $X \oplus \mathbb{R}$.

(2b) $v=2$ and $w=3$. Since $c_{2}^{B}(X) \geq 3>1$, there is a Borel 2-coloring $\chi_{2}: X \rightarrow 2$ witnessing that the singleton $\{a\}$ is not 2-centerpole for Borel colorings of $X$. By the same reason, there is a Borel 2-coloring $\chi_{3}: X \rightarrow 2$ witnessing that the singleton $\{b\}$ is not 2-centerpole for Borel colorings of $X$.

Next consider the (Borel) colorings $\chi_{1}: X \rightarrow 2, \chi_{4}: X \rightarrow 3$, and $\chi_{6}$ : $X \rightarrow 2$ defined by the formulas

$$
\begin{aligned}
& \chi_{1}(x)=1-\chi_{3}\left(a x^{-1} a\right), \\
& \chi_{4}(x)=\min \left(3 \backslash\left\{\chi_{0}\left(a x^{-1} a\right), \chi_{2}\left(b x^{-1} b\right)\right\}\right), \\
& \chi_{6}(x)=\min \left(2 \backslash\left\{\chi_{0}\left(b x^{-1} b\right)\right\}\right) .
\end{aligned}
$$

The (Borel) colorings $\psi$ and $\chi_{t}, t \in\{0,1,2,3,4,6\}$, compose the (Borel) coloring $\tilde{\chi}: X \times \mathbb{R} \rightarrow k$ defined by

$$
\tilde{\chi}(x, t)= \begin{cases}\chi_{t}(x), & \text { if } t \in\{0,1,2,3,4,6\}, \\ \psi(t), & \text { otherwise. }\end{cases}
$$

This coloring $\tilde{\chi}$ witnesses that the set $C \cup A$ is not $k$-centerpole for (Borel) colorings of $X \oplus \mathbb{R}$.

(2c) $v \notin\{1,2\}$. Since $c_{2}^{B}(X)>1$ there is a Borel 2-coloring $\chi_{v}: X \rightarrow 2$ witnessing that the singleton $\{a\}$ is not 2-centerpole for Borel colorings of $X$. By the same reason, there is a Borel 2-coloring $\chi_{w}: X \rightarrow\{1,2\}$ witnessing that the singleton $\{b\}$ is not 2-centerpole for Borel colorings of $X$.

Next, define the (Borel) colorings $\chi_{2 v}, \chi_{2 w}: X \rightarrow 3$ by the formula

$$
\begin{aligned}
& \chi_{2 v}(x)=\min \left(3 \backslash\left\{\chi_{0}\left(a x^{-1} a\right), \psi(2)\right\}\right) \text { and } \\
& \chi_{2 w}(x)=\min \left(2 \backslash\left\{\chi_{0}\left(b x^{-1} b\right)\right\}\right) .
\end{aligned}
$$

Here let us note that the points $2 v$ and 2 are symmetric with respect to $w$ in the group $\mathbb{R}$. 
Finally, define a (Borel) $k$-coloring $\tilde{\chi}: X \oplus \mathbb{R} \rightarrow k$ letting

$$
\tilde{\chi}(x, t)= \begin{cases}\chi_{t}(x) & \text { if } t \in\{0, v, w, 2 v, 2 w\} \\ \psi(t) & \text { otherwise. }\end{cases}
$$

This coloring witnesses that the set $C \cup A$ is not $k$-centerpole for (Borel) colorings of the topological group $X \oplus \mathbb{R}$.

Lemma $7 c_{4}^{B}\left(\mathbb{R}^{m}\right) \geq 8$ for all $m \geq 4$.

Proof This lemma will be proved by induction on $m \geq 4$. For $m=4$ the inequality $c_{4}^{B}\left(\mathbb{R}^{4}\right) \geq t\left(\mathbb{R}^{4}\right)=12 \geq 8$ follows from Lemma 2 . Assume that for some $m \geq 4$ we know that $c_{4}^{B}\left(\mathbb{R}^{m}\right) \geq 8$. The inequality $c_{4}^{B}\left(\mathbb{R}^{m+1}\right) \geq 8$ will follow as soon as we check that each 7-element subset $C \subset \mathbb{R}^{m+1}$ is not 4-centerpole for Borel colorings of $\mathbb{R}^{m+1}$.

Given a 7-element subset $C \subset \mathbb{R}^{m+1}$, find a support $m$-dimensional hyperplane $H \subset \mathbb{R}^{m+1}$ that has at least $\min \{m+1,|C|\} \geq 5$ common points with the set $C$. After a suitable shift, we can assume that the intersection $C \cap H$ contains the origin of $\mathbb{R}^{m+1}$. In this case $H$ is a linear subspace of $\mathbb{R}^{m+1}$ and $\mathbb{R}^{m+1}$ can be written as the direct sum $\mathbb{R}^{m+1}=H \oplus \mathbb{R}$.

Since $|H \cap C| \leq|C| \leq 7$, the inductive assumption guarantees that $H \cap C$ is not 4-centerpole for Borel colorings of $H$. By Lemma $5, c_{3}^{B}\left(\mathbb{R}^{m}\right) \geq 3$. Since $|C \backslash H| \leq 2$, we can apply Lemma 6 and conclude that $C$ is not 4-centerpole for Borel colorings of the topological group $H \oplus \mathbb{R}=\mathbb{R}^{m+1}$.

\section{Centerpole sets in subgroups and groups}

It is clear that each $k$-centerpole subset $C \subset H$ in a subgroup $H$ of a topological group $G$ is $k$-centerpole in $G$. In some cases the converse statement also is true.

Lemma 8 If a subset $C$ of an abelian topological group $G$ is $k$-centerpole in $G$ for some $k \geq 2$, then it is $k$-centerpole in the subgroup $H=\langle C\rangle+G[2]$.

Proof Observe that for each $x \in G \backslash H$ the cosets $c+2\langle C\rangle$ and $-x+2\langle C\rangle$ are disjoint. Assuming the opposite, we would conclude that $2 x \in 2\langle C\rangle$ and hence $x \in$ $\langle C\rangle+G[2]=H$, which contradicts the choice of $x$.

Now we are able to prove that the set $C$ is $k$-centerpole in the group $H$. Given any $k$-coloring $\chi: H \rightarrow k$, extend $\chi$ to a $k$-coloring $\tilde{\chi}: G \rightarrow k$ such that for each $x \in G \backslash H$ the coset $x+2\langle C\rangle$ is monochromatic and its color is different from the color of the coset $-x+2\langle C\rangle$.

Since $C$ is $k$-centerpole in the group $G$, there is an unbounded monochromatic subset $S \subset G$ such that $S=2 c-S$ for some $c \in C$. We claim that $S \subset H$. Assuming the converse, we would find a point $x \in S \backslash H$ and conclude that the coset $x+2\langle C\rangle$ 
has the same color as the coset $2 c-x+2\langle C\rangle=-x+2\langle C\rangle$, which contradicts the choice of the coloring $\tilde{\chi}$.

The Borel version of this result is a bit more difficult.

Lemma 9 Let $k \geq 2$ and $H$ be a Borel subgroup of an abelian topological group $G$ such that $G[2] \subset H$. A subset $C \subset H$ is $k$-centerpole for Borel colorings of $H$ if $C$ is $k$-centerpole for Borel colorings of $G$, the subgroup $2 H=\{2 x: x \in H\}$ is closed in $G$, and the subspace $X=(G / 2 H) \backslash(H / 2 H)$ contains a Borel subset $B$ that has one-point intersection with each set $\{x,-x\}, x \in X$. Such a Borel set $B \subset X$ exists if the space $X$ is paracompact.

Proof Given any Borel $k$-coloring $\chi: H \rightarrow k$, extend $\chi$ to a Borel $k$-coloring $\tilde{\chi}$ : $G \rightarrow k$ defined by

$$
\tilde{\chi}(x)= \begin{cases}\chi(x), & \text { if } x \in H, \\ 0, & \text { if } x \in G \backslash H \text { and } x+2 H \in B, \\ 1, & \text { if } x \in G \backslash H \text { and } x+2 H \notin B .\end{cases}
$$

Since $C$ is $k$-centerpole for Borel colorings of the group $G$, there is an unbounded monochromatic subset $S \subset G$, symmetric with respect to some point $c \in C$. We claim that $S \subset H$, witnessing that $C$ is $k$-centerpole for Borel colorings of $H$.

Assuming conversely that $S \not \subset H$, find a point $x \in S \backslash H$. It follows that $x$ and $2 c-x$ have the same color. If this color is 0 , then the cosets $x+2 H$ and $2 c-x+$ $2 H=-x+2 H=-(x+2 H)$ both belong to the set $B \subset G / 2 H$. By our hypothesis $B$ has one-point intersection with the set $\{x+2 H,-(x+2 H)\}$. Consequently, $x+$ $2 H=-(x+2 H)$ and hence $2 x \in 2 H$ and $x \in H+G[2]=H$, which contradicts the choice of the point $x$. If the color of the cosets $x+2 H$ and $2 c-x+2 H=-(x+2 H)$ is 1 , then $(x+2 H),-(x+2 H) \notin B$ and then $x+2 H=-(x+2 H)$ because $B$ has one-point intersection with the set $\{x+2 H,-(x+2 H)\}$. This again leads to a contradiction.

Claim 16 If the space $X=(G / 2 H) \backslash(H / 2 H)$ is paracompact, then $X$ contains a Borel subset $B \subset X$ that has one-point intersection with each set $\{x,-x\}, x \in X$.

Consider the action

$$
\alpha: C_{2} \times X \rightarrow X, \quad \alpha:(\varepsilon, x) \mapsto \varepsilon \cdot x,
$$

of the cyclic group $C_{2}=\{1,-1\}$ on the space $X$ and let $X / C_{2}=\{\{x,-x\}: x \in X\}$ be the orbit space of this action. It is easy to check that the orbit map $q: X \rightarrow X / C_{2}$ is closed and then the orbit space $X / C_{2}$ is paracompact as the image of a paracompact space under a closed map, see Michael, Theorem 5.1.33 in [7].

Since $H \supset 2 H+G[2]$, for every $x \in G \backslash H$ the cosets $x+2 H$ and $-x+2 H$ are disjoint, which implies that each point $x \in X$ is distinct from $-x$. Then each point $x \in X$ has a neighborhood $U_{x} \subset X$ such that $U_{x} \cap-U_{x}=\emptyset$. Replacing $U_{x}$ 
by $U_{x} \cap\left(-U_{-x}\right)$ we can additionally assume that $U_{x}=-U_{-x}$. Now consider the open neighborhood $U_{ \pm x}=q\left(U_{x}\right)=q\left(U_{-x}\right) \subset X / C_{2}$ of the orbit $\{x,-x\} \in X / C_{2}$ of the point $x \in X$. By the paracompactness of $X / C_{2}$ the open cover $\left\{U_{ \pm x}: x \in X\right\}$ of $X / C_{2}$ has a $\Sigma$-discrete refinement $\mathcal{U}=\bigcup_{n \in \omega} \mathcal{U}_{n}$. This means that each family $\mathcal{U}_{n}$, $n \in \omega$, is discrete in $X / C_{2}$. For each $U \in \mathcal{U}$ find a point $x_{U} \in X$ such that $U \subset U_{ \pm x_{U}}$. For every $n \in \omega$ consider the open subset $W_{n}=\bigcup_{U \in \mathcal{U}_{n}} q^{-1}(U) \cap U_{x_{U}}$ of the space $X$ and let $\pm W_{n}=-W_{n} \cup W_{n}$. One can check that the Borel subset

$$
B=\bigcup_{n \in \omega}\left(W_{n} \backslash \bigcup_{i<n} \pm W_{i}\right)
$$

of $X$ has one-point intersection with each orbit $\{x,-x\}, x \in X$.

The following lemma will be helpful in the proof of the upper bound $r c_{k}^{B}(G) \leq$ $c_{k}^{B}(G)-2$ from Proposition 1.

Lemma 10 Let $k \geq 4$ and $C \subset \mathbb{R}^{\omega}$ be a finite $k$-centerpole subset for Borel colorings of $\mathbb{R}^{\omega}$. Then the affine hull of $C$ in $\mathbb{R}^{\omega}$ has dimension $\leq|C|-3$.

Proof This lemma will be proved by induction on the cardinality $|C|$.

First observe that $|C| \geq c_{k}^{B}\left(\mathbb{R}^{\omega}\right) \geq c_{3}^{B}\left(\mathbb{R}^{\omega}\right) \geq 6$ by Lemma 5 . So, we start the induction with $|C|=6$.

Suppose that either $m=6$ or $m>6$ and the lemma is true for all $C$ with $6 \leq|C|<$ $m$. Fix a $k$-centerpole subset $C \subset \mathbb{R}^{\omega}$ for Borel colorings of cardinality $|C|=m$. We need to show that the affine hull $A$ of $C$ has dimension $\operatorname{dim} A \leq m-3$. Assuming the opposite, we can find a support hyperplane $H \subset A$ for $C$ such that $|H \cap C| \geq$ $\operatorname{dim} H+1=\operatorname{dim} A \geq|C|-2$ and hence $0<|C \backslash H| \leq 2$. After a suitable shift, we can assume that $H$ contains the origin of $\mathbb{R}^{\omega}$ and hence is a subgroup of $\mathbb{R}^{\omega}$. In this case the affine hull $A$ is a linear subspace in $\mathbb{R}^{\omega}$ that can be identified with the direct sum $H \oplus \mathbb{R}$. It follows that $\operatorname{dim} H=\operatorname{dim} A-1 \geq|C|-2-1 \geq|C \cap H|-2$.

We claim that the set $H \cap C$ is not $k$-centerpole for Borel colorings of the topological group $H$.

If $6 \leq|C \cap H|<|C|=m$, then by the inductive assumption, the set $C \cap H$ is not $k$-centerpole for Borel colorings of $\mathbb{R}^{\omega}$ because its affine hull $H$ has dimension $\operatorname{dim} H \geq|C \cap H|-2$. If $|C \cap H|<6$ (which happens for $m=6$ ), then the inequalities $c_{k}^{B}(H) \geq c_{3}^{B}(H) \geq 6=m=|C|>|H \cap C|$ given by Lemma 5 guarantee that $C \cap H$ is not $k$-centerpole for Borel colorings of $\mathbb{R}^{\omega}$.

By (the proof) of Proposition 1 in [3], $c_{2}^{B}(H)=3$. Since $H$ is a support hyperplane for $C$ and $|C \backslash H| \leq 2$, we can apply Lemma 6 and conclude that $C$ is not $k$-centerpole for Borel colorings of $H \oplus \mathbb{R}=A$. Since the subgroup $2 A$ is closed in the metrizable group $\mathbb{R}^{\omega}$, by Lemma $9, C$ is not $k$-centerpole for Borel colorings of $\mathbb{R}^{\omega}$ and this is a desired contradiction that completes the proof of the inductive step and base of the induction.

\section{Stability properties}

In this section we shall prove some particular cases of the Stability Theorem 4. 
Lemma 11 For any numbers $k \geq 2$ and $n \leq m$

$$
c_{k}^{B}\left(\mathbb{R}^{n} \times \mathbb{Z}^{m-n}\right)= \begin{cases}c_{k}^{B}\left(\mathbb{R}^{n} \times \mathbb{Z}^{\omega}\right), & \text { if } m \geq r c_{k}^{B}\left(\mathbb{R}^{n} \times \mathbb{Z}^{\omega}\right), \\ c_{k}^{B}\left(\mathbb{R}^{\omega}\right), & \text { if } n \geq r c_{k}^{B}\left(\mathbb{R}^{\omega}\right) .\end{cases}
$$

Proof First assume that $m \geq r c_{k}^{B}\left(\mathbb{R}^{n} \times \mathbb{Z}^{\omega}\right)$. By the definition of the number $r=$ $r c_{k}^{B}\left(\mathbb{R}^{n} \times \mathbb{Z}^{\omega}\right)$, the topological group $G=\mathbb{R}^{n} \times \mathbb{Z}^{\omega}$ contains a $k$-centerpole subset $C \subset G$ of cardinality $|C|=c_{k}^{B}(G)$ that generates a subgroup $\langle C\rangle \subset \mathbb{Z}^{\omega}$ of $\mathbb{Z}$-rank $r$. It follows that the linear subspace $L \subset \mathbb{R}^{n} \times \mathbb{R}^{\omega}$ generated by the set $C$ has dimension $r$. Then $H=L \cap G$, being a closed subgroup of $\mathbb{Z}$-rank $r$ in the $r$-dimensional vector space $L$ is topologically isomorphic to $\mathbb{R}^{s} \times \mathbb{Z}^{r-s}$ for some $s \leq r \leq m$, see Theorem 6 in [10]. Taking into account that $H$ is a closed subgroup of $G=\mathbb{R}^{n} \times \mathbb{Z}^{\omega}$, we conclude that $s \leq n$. By Lemma 9 , the set $C$ is $k$-centerpole in $H$ for Borel colorings. Consequently,

$$
\begin{aligned}
c_{k}^{B}\left(\mathbb{R}^{n} \times \mathbb{Z}^{\omega}\right) & \leq c_{k}^{B}\left(\mathbb{R}^{n} \times \mathbb{Z}^{m-n}\right) \leq c_{k}^{B}\left(\mathbb{R}^{s} \times \mathbb{Z}^{r-s}\right)=c_{k}^{B}(H) \leq|C|=c_{k}^{B}(G) \\
& =c_{k}^{B}\left(\mathbb{R}^{n} \times \mathbb{Z}^{\omega}\right)
\end{aligned}
$$

implies the desired equality $c_{k}^{B}\left(\mathbb{R}^{n} \times \mathbb{Z}^{m-n}\right)=c_{k}^{B}\left(\mathbb{R}^{n} \times \mathbb{Z}^{\omega}\right)$.

Now assume that $n \geq r c_{k}^{B}\left(\mathbb{R}^{\omega}\right)$. In this case we can repeat the above argument for a set $C \subset \mathbb{R}^{\omega}$ of cardinality $|C|=c_{k}^{B}\left(\mathbb{R}^{\omega}\right)$ that generates a subgroup $\langle C\rangle \subset \mathbb{R}^{\omega}$ of $\mathbb{Z}$-rank $r=r c_{k}^{B}\left(\mathbb{R}^{\omega}\right)$. Then the linear subspace $L \subset \mathbb{R}^{\omega}$ generated by the set $C$ is topologically isomorphic to $\mathbb{R}^{r}$. By Lemma 9 , the set $C$ is $k$-centerpole for Borel colorings of $L$. Since $\mathbb{R}^{r} \hookrightarrow \mathbb{R}^{n} \times \mathbb{Z}^{m-n} \hookrightarrow \mathbb{R}^{\omega}$, we get

$$
c_{k}^{B}\left(\mathbb{R}^{\omega}\right) \leq c_{k}^{B}\left(\mathbb{R}^{n} \times \mathbb{Z}^{m-n}\right) \leq c_{k}^{B}\left(\mathbb{R}^{r}\right)=c_{k}^{B}(L) \leq|C|=c_{k}^{B}\left(\mathbb{R}^{\omega}\right)
$$

and hence $c_{k}^{B}\left(\mathbb{R}^{n} \times \mathbb{Z}^{m-n}\right)=c_{k}^{B}\left(\mathbb{R}^{\omega}\right)$.

Lemma $12 c_{k}\left(\mathbb{R}^{n} \times \mathbb{Z}^{m-n}\right)=c_{k}^{B}\left(\mathbb{Z}^{\omega}\right)$ for any numbers $k \in \mathbb{N}$ and $n \leq m$ with $m \geq$ $c_{k}^{B}\left(\mathbb{Z}^{\omega}\right)$.

Proof For $k=1$ the equality $c_{k}\left(\mathbb{R}^{n} \times \mathbb{Z}^{m-n}\right)=1=c_{k}^{B}\left(\mathbb{Z}^{\omega}\right)$ is trivial. So we assume that $k \geq 2$.

We claim that $c_{k}^{B}\left(\mathbb{Z}^{\omega}\right) \leq c_{k}\left(\mathbb{R}^{m}\right)$. Indeed, take any $k$-centerpole subset $C \subset \mathbb{R}^{\omega}$ of cardinality $|C|=c_{k}\left(\mathbb{R}^{m}\right)$. By Lemma 8 , the set $C$ is $k$-centerpole in the subgroup $\langle C\rangle \subset \mathbb{R}^{\omega}$ generated by $C$. Being a torsion-free finitely generated abelian group, $\langle C\rangle$ is algebraically isomorphic to $\mathbb{Z}^{r}$ for some $r \in \omega$. Then

$$
c_{k}\left(\mathbb{Z}^{r}\right) \leq c_{k}(\langle C\rangle) \leq|C|=c_{k}\left(\mathbb{R}^{m}\right) .
$$

On the other hand, Lemma 11 ensures that

$$
c_{k}\left(\mathbb{R}^{m}\right) \leq c_{k}\left(\mathbb{Z}^{m}\right)=c_{k}^{B}\left(\mathbb{Z}^{m}\right)=c_{k}^{B}\left(\mathbb{Z}^{\omega}\right) .
$$


Unifying these inequalities we get

$$
\begin{aligned}
c_{k}^{B}\left(\mathbb{Z}^{\omega}\right) & \leq c_{k}^{B}\left(\mathbb{Z}^{r}\right)=c_{k}\left(\mathbb{Z}^{r}\right) \leq c_{k}\left(\mathbb{R}^{m}\right) \leq c_{k}\left(\mathbb{R}^{n} \times \mathbb{Z}^{m-n}\right) \leq c_{k}\left(\mathbb{Z}^{m}\right)=c_{k}^{B}\left(\mathbb{Z}^{m}\right) \\
& =c_{k}^{B}\left(\mathbb{Z}^{\omega}\right),
\end{aligned}
$$

which implies the desired equality $c_{k}\left(\mathbb{R}^{n} \times \mathbb{Z}^{m-n}\right)=c_{k}^{B}\left(\mathbb{Z}^{\omega}\right)$.

\section{Proof of Theorem 3}

1. The upper bound $c_{k}\left(\mathbb{Z}^{n}\right) \leq c_{k}\left(\mathbb{Z}^{k}\right) \leq 2^{k}-1-\max _{s \leq k-2}\left(\begin{array}{l}k-1 \\ s-1\end{array}\right)$ for $k \leq n$ follows from Theorem 5.

2. By Proposition 3 and Theorem $6(7), c_{n}\left(\mathbb{Z}^{n}\right) \geq c_{n}\left(\mathbb{R}^{n}\right) \geq c_{n}^{B}\left(\mathbb{R}^{n}\right) \geq t\left(\mathbb{R}^{n}\right) \geq$ $\frac{1}{2}\left(n^{2}+3 n-4\right)$.

For technical reasons, first we prove the statement (4) of Theorem 3 and after that return back to the statement (3).

4. Let $1 \leq k \leq m \leq \omega$ be two numbers. We need to prove that $c_{k}^{B}\left(\mathbb{R}^{m}\right)<$ $c_{k+1}^{B}\left(\mathbb{R}^{m+1}\right)$ and $c_{k}\left(\mathbb{R}^{m}\right)<c_{k+1}\left(\mathbb{R}^{m+1}\right)$.

First we assume that $m$ is finite. The strict inequality $c_{k}^{B}\left(\mathbb{R}^{m}\right)<c_{k+1}^{B}\left(\mathbb{R}^{m+1}\right)$ will follow as soon as we show that any subset $C \subset \mathbb{R}^{m+1}$ of cardinality $|C| \leq c_{k}^{B}\left(\mathbb{R}^{m}\right)$ fails to be $(k+1)$-centerpole for Borel colorings of $\mathbb{R}^{m+1}$. If $C$ is a singleton, then it is not $(k+1)$-centerpole since $c_{k+1}^{B}\left(\mathbb{R}^{m+1}\right) \geq c_{2}^{B}\left(\mathbb{R}^{m+1}\right) \geq 3$ by (the proof of) Proposition 4.1 in [3]. So, $C$ contains two distinct points $a, b$. Let $L=\mathbb{R} \cdot(a-$ b) $\subset \mathbb{R}^{m+1}$ be the linear subspace generated by the vector $a-b$. Write the space $\mathbb{R}^{m+1}$ as the direct sum $\mathbb{R}^{m+1}=H \oplus L$ where $H$ is a linear $m$-dimensional subspace of $\mathbb{R}^{m+1}$ and consider the projection pr: $\mathbb{R}^{m+1} \rightarrow H$ whose kernel is equal to $L$. Since $\operatorname{pr}(a)=\operatorname{pr}(b)$, the projection of the set $C$ onto the subspace $H$ has cardinality $|\operatorname{pr}(C)|<|C| \leq c_{k}^{B}\left(\mathbb{R}^{m}\right)=c_{k}^{B}(H)$ and hence $\operatorname{pr}_{H}(C)$ is not $k$-centerpole for Borel $k$-colorings of the group $H$. Consequently, there is a Borel $k$-coloring $\chi: H \rightarrow k$ such that no monochromatic unbounded subset of $H$ is symmetric with respect to a point $c \in \operatorname{pr}(C)$.

For a real number $\gamma \in \mathbb{R}$, consider the half-line $L_{\gamma}^{+}=\{t(a-b): t \geq \gamma\}$ of $L$. Since the subset $C \subset \mathbb{R}^{m+1}$ is finite, there is $\gamma \in \mathbb{R}$ such that $C \subset H+L_{\gamma}^{+}$.

Now define a Borel $(k+1)$-coloring $\tilde{\chi}: H \oplus L \rightarrow k+1=\{0, \ldots, k\}$ by the formula

$$
\tilde{\chi}(x)= \begin{cases}\chi(\operatorname{pr}(x)), & \text { if } x \in H+L_{\gamma}^{+}, \\ k, & \text { otherwise. }\end{cases}
$$

It can be shown that this coloring witnesses that $C$ is not $(k+1)$-centerpole for Borel colorings of $\mathbb{R}^{m+1}=H \oplus L$.

Now assume that the number $m$ is infinite. Then for the finite number $r=$ $\max \left\{r c_{k}^{B}\left(\mathbb{R}^{\omega}\right), r c_{k+1}^{B}\left(\mathbb{R}^{\omega}\right)\right\}$ we get $c_{k}^{B}\left(\mathbb{R}^{r}\right)=c_{k}^{B}\left(\mathbb{R}^{\omega}\right)$ and $c_{k+1}^{B}\left(\mathbb{R}^{r+1}\right)=c_{k+1}^{B}\left(\mathbb{R}^{\omega}\right)$ by the stabilization Lemma 11. Since $r$ is finite, the case considered above guarantees that

$$
c_{k}^{B}\left(\mathbb{R}^{m}\right)=c_{k}^{B}\left(\mathbb{R}^{m}\right)=c_{k}^{B}\left(\mathbb{R}^{r}\right)<c_{k+1}^{B}\left(\mathbb{R}^{r+1}\right)=c_{k+1}^{B}\left(\mathbb{R}^{\omega}\right)=c_{k+1}\left(\mathbb{R}^{m+1}\right) .
$$


By analogy we can prove the strict inequality $c_{k}\left(\mathbb{R}^{m}\right)<c_{k}\left(\mathbb{R}^{m+1}\right)$.

3. Now we are able to prove the lower bound $c_{k}^{B}\left(\mathbb{R}^{\omega}\right) \geq k+4$ from the statement (3) of Theorem 3. By the preceding item, $c_{k+1}^{B}\left(\mathbb{R}^{\omega}\right) \geq 1+c_{k}^{B}\left(\mathbb{R}^{\omega}\right)$ for all $k \in \mathbb{N}$. By induction, we shall show that $c_{k}^{B}\left(\mathbb{R}^{\omega}\right) \geq k+4$ for all $k \geq 4$. For $k=4$ the inequality $c_{4}^{B}\left(\mathbb{R}^{\omega}\right) \geq 8 \geq 4+4$ was proved in Lemma 7 . Assuming that $c_{k}^{B}\left(\mathbb{R}^{\omega}\right) \geq k+4$ for some $k \geq 4$, we conclude that $c_{k+1}^{B}\left(\mathbb{R}^{\omega}\right)>c_{k}^{B}\left(\mathbb{R}^{\omega}\right) \geq k+4$ and hence $c_{k+1}^{B}\left(\mathbb{R}^{\omega}\right) \geq$ $(k+1)+4$.

Now we see that for every $n \geq k \geq 4$ we have the desired lower bound:

$$
c_{k}^{B}\left(\mathbb{R}^{n}\right) \geq c_{k}^{B}\left(\mathbb{R}^{\omega}\right) \geq k+4 .
$$

5. Let $k \in \mathbb{N}$ and $n, m \in \omega \cup\{\omega\}$ be numbers with $1 \leq k \leq n+m$. We need to prove that $c_{k}^{B}\left(\mathbb{R}^{n} \times \mathbb{Z}^{m}\right)<c_{k+1}^{B}\left(\mathbb{R}^{n} \times \mathbb{Z}^{m+1}\right)$ and $c_{k}\left(\mathbb{R}^{n} \times \mathbb{Z}^{m}\right)<c_{k+1}\left(\mathbb{R}^{n} \times \mathbb{Z}^{m+1}\right)$. According to the Stabilization Lemma 11, it suffices to consider the case of finite numbers $n, m$.

First we prove the inequality $c_{k}^{B}\left(\mathbb{R}^{n} \times \mathbb{Z}^{m}\right)<c_{k+1}^{B}\left(\mathbb{R}^{n} \times \mathbb{Z}^{m+1}\right)$. We need to show that each subset $C \subset \mathbb{R}^{n} \times \mathbb{Z}^{m+1}$ of cardinality $|C| \leq c_{k}^{B}\left(\mathbb{R}^{n} \times \mathbb{Z}^{m}\right)$ is not $(k+1)$ centerpole in $\mathbb{R}^{n} \times \mathbb{Z}^{m+1}$ for Borel colorings. We shall identify $\mathbb{R}^{n} \times \mathbb{Z}^{m+1}$ with the direct sum $\mathbb{R}^{n} \oplus \mathbb{Z}^{m+1}$. Since $k \leq n+m$, Theorem 5 implies that the numbers $|C| \leq c_{k}^{B}\left(\mathbb{R}^{n} \times \mathbb{Z}^{m}\right) \leq c_{k}\left(\mathbb{Z}^{n+m}\right) \leq c_{k}\left(\mathbb{Z}^{k}\right)$ all are finite.

Three cases are possible.

(i) $|C| \leq 1$. In this case we can assume that $C=\{0\}$ and take any coloring $\chi$ : $\mathbb{R}^{n} \oplus \mathbb{Z}^{m+1} \rightarrow k+1$ such that the color of each non-zero element $x \in \mathbb{R}^{n} \times \mathbb{Z}^{m+1}$ differs from the color of $-x$. This coloring witnesses that $C$ is not $(k+1)$ centerpole in $\mathbb{R}^{n} \times \mathbb{Z}^{m+1}$.

(ii) $|C|>1$ and $C \subset z+\mathbb{R}^{n}$ for some $z \in \mathbb{Z}^{m+1}$. Without lose of generality, $z=$ 0 and hence $C \subset \mathbb{R}^{n}$. Take two distinct points $a, b \in C$ and consider the 1dimensional linear subspace $L=\mathbb{R} \cdot(a-b) \subset \mathbb{R}^{n}$ generated by the vector $a-b$. Write the space $\mathbb{R}^{n}$ as the direct sum $\mathbb{R}^{n}=L \oplus H$ where $H$ is a linear $(n-1)$ dimensional subspace of $\mathbb{R}^{n}$ and consider the projection pr: $\mathbb{R}^{n} \oplus \mathbb{Z}^{m+1} \rightarrow H \oplus$ $\mathbb{Z}^{m+1}$ whose kernel is equal to $L$. Since $\operatorname{pr}(a)=\operatorname{pr}(b)$, the projection of the set $C$ onto the subgroup $H \oplus \mathbb{Z}^{m+1}$ of $\mathbb{R}^{n} \oplus \mathbb{Z}^{m+1}$ has cardinality

$$
|\operatorname{pr}(C)|<|C| \leq c_{k}^{B}\left(\mathbb{R}^{n} \times \mathbb{Z}^{m}\right) \leq c_{k}^{B}\left(\mathbb{R}^{n-1} \times \mathbb{Z}^{m+1}\right)=c_{k}^{B}\left(H \oplus \mathbb{Z}^{m+1}\right)
$$

and hence $\operatorname{pr}_{H}(C)$ is not $k$-centerpole for Borel colorings of the group $H \oplus$ $\mathbb{Z}^{m+1}$. Consequently, there is a Borel $k$-coloring $\chi: H \oplus \mathbb{Z}^{m+1} \rightarrow k$ such that no monochromatic unbounded subset of $H \oplus \mathbb{Z}^{m+1}$ is symmetric with respect to a point $c \in \operatorname{pr}(C)$.

For a real number $\gamma \in \mathbb{R}$, consider the half-line $L_{\gamma}^{+}=\{t(a-b): t \geq \gamma\}$ of $L$. Since the subset $C \subset \mathbb{R}^{n} \oplus \mathbb{Z}^{m+1}=H \oplus L \oplus \mathbb{Z}^{m+1}$ is finite, there is $\gamma \in \mathbb{R}$ such that $C \subset H+L_{\gamma}^{+}+\mathbb{Z}^{m+1}$.

Now define a Borel $(k+1)$-coloring $\tilde{\chi}: H \oplus L \oplus \mathbb{Z}^{m+1} \rightarrow k+1=\{0, \ldots, k\}$ by the formula

$$
\tilde{\chi}(x)= \begin{cases}\chi(\operatorname{pr}(x)), & \text { if } x \in H+L_{\gamma}^{+}+\mathbb{Z}^{m+1}, \\ k, & \text { otherwise. }\end{cases}
$$


It can be shown that this coloring witnesses that $C$ is not $(k+1)$-centerpole for Borel colorings of $\mathbb{R}^{n} \oplus \mathbb{Z}^{m+1}=H \oplus L \oplus \mathbb{Z}^{m+1}$.

(iii) The set $C \subset \mathbb{R}^{n} \oplus \mathbb{Z}^{m+1}$ contains two points $a, b$ whose projections on the subspace $\mathbb{Z}^{m+1}$ are distinct. Without loss of generality, the projections of $a, b$ on the last coordinate are distinct. Then the 1 -dimensional subspace $L=\mathbb{R} \cdot(a-b)$ of $\mathbb{R}^{n} \times \mathbb{R}^{m+1}$ meets the subspace $\mathbb{R}^{n} \oplus \mathbb{R}^{m}$ and hence $\mathbb{R}^{n} \oplus \mathbb{R}^{m+1}$ can be identified with the direct sum $\mathbb{R}^{n} \oplus \mathbb{R}^{m} \oplus L$. Let pr : $\mathbb{R}^{n} \times \mathbb{R}^{m+1} \rightarrow \mathbb{R}^{n} \times \mathbb{R}^{m}$ be the projection whose kernel coincides with $L$. Since pr is an open map, the image $H=\operatorname{pr}\left(\mathbb{R}^{n} \times \mathbb{Z}^{m+1}\right.$ ) is a locally compact (and hence closed) subgroup of $\mathbb{R}^{n} \times \mathbb{R}^{m}$, which can be written as the countable union of shifted copies of the space $\mathbb{R}^{n}$. By Theorem 6 of [10], $H$ is topologically isomorphic to $\mathbb{R}^{n} \times \mathbb{Z}^{m}$. It follows from the definition of $H$ that $\mathbb{R}^{n} \oplus \mathbb{Z}^{m+1} \subset H \oplus L$.

Since $\operatorname{pr}(a)=\operatorname{pr}(b)$, the projection of the set $C$ has cardinality $|\operatorname{pr}(C)|<$ $|C| \leq c_{k}^{B}\left(\mathbb{R}^{n} \oplus \mathbb{Z}^{m}\right)=c_{k}^{B}(H)$, which means that $\operatorname{pr}(C)$ is not $k$-centerpole for Borel colorings of $H$. Consequently, there is a Borel $k$-coloring $\chi: H \rightarrow k$ such that no monochromatic unbounded subset of $H$ is symmetric with respect to a point $c \in \operatorname{pr}(C)$.

For a real number $\gamma \in \mathbb{R}$, consider the half-line $L_{\gamma}^{+}=\{t(a-b): t \geq \gamma\}$ of $L$. Since the subset $C \subset H \oplus L$ is finite, there is $\gamma \in \mathbb{R}$ such that $C \subset H+L_{\gamma}^{+}$.

Now define a Borel $(k+1)$-coloring $\tilde{\chi}: H \oplus L \rightarrow k+1$ by the formula

$$
\tilde{\chi}(x)= \begin{cases}\chi(\operatorname{pr}(x)), & \text { if } x \in H+L_{\gamma}^{+}, \\ k, & \text { otherwise }\end{cases}
$$

It can be shown that this coloring witnesses that $C$ is not $(k+1)$-centerpole for Borel colorings of $H \oplus L \supset \mathbb{R}^{n} \oplus \mathbb{Z}^{m+1}$.

After considering these three cases, we can conclude that $c_{k+1}^{B}\left(\mathbb{R}^{n} \times \mathbb{Z}^{m+1}\right)>$ $c_{k}^{B}\left(\mathbb{R}^{n} \times \mathbb{Z}^{m}\right)$.

Deleting the adjective "Borel" from the above proof, we get the proof of the strict inequality

$$
c_{k}\left(\mathbb{R}^{n} \times \mathbb{Z}^{m}\right)<c_{k+1}\left(\mathbb{R}^{n} \times \mathbb{Z}^{m+1}\right) .
$$

\section{Proof of Theorem 2}

In this section we prove Theorem 2. Let $k, n, m$ be cardinals. We shall use known upper bounds for the numbers $c_{k}\left(\mathbb{Z}^{n}\right)$, lower bounds for $t\left(\mathbb{R}^{n}\right)$ and the inequality

$$
t\left(\mathbb{R}^{n+m}\right) \leq c_{k}^{B}\left(\mathbb{R}^{n+m}\right) \leq c_{k}^{B}\left(\mathbb{R}^{n} \times \mathbb{Z}^{m}\right) \leq c_{k}\left(\mathbb{R}^{n} \times \mathbb{Z}^{m}\right) \leq c_{k}\left(\mathbb{Z}^{m}\right)
$$

established in Proposition 3.

1. Assume that $n+m \geq 1$. Since each singleton is 1-centerpole for (Borel) colorings of the group $\mathbb{R}^{n} \times \mathbb{Z}^{m}$, we conclude that $c_{1}\left(\mathbb{R}^{n} \times \mathbb{Z}^{m}\right)=c_{1}^{B}\left(\mathbb{R}^{n} \times \mathbb{Z}^{m}\right)=1$. 
2. Assume that $n+m \geq 2$. The inequalities $3 \leq t\left(\mathbb{R}^{2}\right) \leq c_{2}^{B}\left(\mathbb{R}^{2}\right) \leq c_{2}\left(\mathbb{Z}^{2}\right) \leq 3$ follow from Theorem 5, 6(2) and Proposition 3.

We claim that $c_{2}^{B}\left(\mathbb{R}^{\omega}\right) \geq 3$. Assuming that $c_{2}^{B}\left(\mathbb{R}^{\omega}\right)<3$ we conclude that $r c_{k}^{B}\left(\mathbb{R}^{\omega}\right) \leq c_{2}^{B}\left(\mathbb{R}^{\omega}\right)-1 \leq 1$. Then by the Stabilization Lemma 11 , we get that $c_{2}\left(\mathbb{R}^{1}\right)=c_{2}\left(\mathbb{R}^{\omega}\right)$ is finite. On the other hand, the real line has the 2-coloring $\chi: \mathbb{R} \rightarrow 2, \chi^{-1}(1)=(0, \infty)$, without unbounded monochromatic symmetric subsets. This coloring witnesses that $c_{2}\left(\mathbb{R}^{1}\right)=\infty$ and this is a contradiction. Therefore,

$$
3 \leq c_{2}^{B}\left(\mathbb{R}^{\omega}\right) \leq c_{2}^{B}\left(\mathbb{R}^{n} \times \mathbb{Z}^{m-n}\right) \leq c_{2}^{B}\left(\mathbb{R}^{n} \times \mathbb{Z}^{m-n}\right) \leq c_{2}\left(\mathbb{Z}^{2}\right)=3
$$

3. Assume that $n+m \geq 3$. Lemma 5 and Theorem 5 imply the inequalities

$$
6 \leq c_{3}^{B}\left(\mathbb{R}^{m}\right) \leq c_{3}^{B}\left(\mathbb{R}^{n} \times \mathbb{Z}^{m-n}\right) \leq c_{3}^{B}\left(\mathbb{R}^{n} \times \mathbb{Z}^{m-n}\right) \leq c_{3}\left(\mathbb{Z}^{3}\right)=6
$$

that turn into equalities.

4. Assume that $n+m=4$. Theorem 5, 6(4) and Proposition 3 imply the inequalities

$$
12 \leq t\left(\mathbb{R}^{4}\right) \leq c_{4}^{B}\left(\mathbb{R}^{4}\right) \leq c_{4}^{B}\left(\mathbb{R}^{n} \times \mathbb{Z}^{m}\right) \leq c_{4}\left(\mathbb{R}^{n} \times \mathbb{Z}^{m}\right) \leq c_{4}\left(\mathbb{Z}^{4}\right) \leq 12,
$$

which actually are equalities.

5. We need to prove that $c_{k}^{B}\left(\mathbb{R}^{n} \times \mathbb{Z}^{m}\right)=\infty$ if $k \geq n+m+1<\omega$. This equality will follow as soon as we check that $c_{k}^{B}\left(\mathbb{R}^{n+m}\right)=\infty$. Let $\Delta$ be a simplex in $\mathbb{R}^{n+m}$ centered at the origin. Write the boundary $\partial \Delta$ as the union $\partial \Delta=\bigcup_{i=0}^{n+m} \Delta_{i}$ of its facets. Define a Borel $k$-coloring $\chi: \mathbb{R}^{n} \rightarrow\{0, \ldots, n+m\} \subset k$ assigning to each point $x \in \mathbb{R}^{n} \backslash\{0\}$ the smallest number $i \leq n+m$ such that the ray $\mathbb{R}_{+} \cdot x$ meets the facet $\Delta_{i}$. Also put $\chi(0)=0$. It is easy to check that the coloring $\chi$ witnesses that the set $\mathbb{R}^{n+m}$ is not $k$-centerpole for Borel colorings of $\mathbb{R}^{n+m}$ and consequently, $c_{k}^{B}\left(\mathbb{R}^{n+m}\right)=\infty$.

6. Assuming that $k \geq n+m+1$, we shall show that $c_{k}\left(\mathbb{R}^{n} \times \mathbb{Z}^{m}\right)=\infty$. If $n+m$ is finite, then this follows from the preceding item. So, we assume that $n+m$ is infinite. Then the group $G=\mathbb{R}^{n} \times \mathbb{Z}^{m}$ has cardinality $2^{n+m}$. By Theorem 4 of [4], for the group $G$ endowed with the discrete topology, we get $\nu(G)=\log |G|=$ $\min \left\{\gamma: 2^{\gamma} \geq|G|\right\} \leq n+m \leq k$, which means that $G$ admits a $k$-coloring without infinite monochromatic symmetric subset. This implies that the set $G$ is not $k$ centerpole in $G$ and thus $c_{k}(G)=\infty$.

7. Assume that $n+m \geq \omega$ and $\omega \leq k<\operatorname{cov}(\mathcal{M})$. The lower bound from Theorem 3(3) implies that $\omega \leq c_{k}^{B}\left(\mathbb{R}^{\omega}\right) \leq c_{k}^{B}\left(\mathbb{Z}^{\omega}\right)$. The upper bound $c_{\kappa}^{B}\left(\mathbb{Z}^{\omega}\right) \leq \omega$ will follow as soon as we check that each countable dense subset $C \subset \mathbb{Z}^{\omega}$ is $\kappa$-centerpole for Borel colorings of $\mathbb{Z}^{\omega}$. Let $\chi: \mathbb{Z}^{\omega} \rightarrow \kappa$ be a Borel $\kappa$-coloring of $\mathbb{Z}^{\omega}$. Taking into account that $\mathbb{Z}^{\omega}=\bigcup_{i \in \kappa} \chi^{-1}(i)$ is homeomorphic to a dense $G_{\delta}$-subset of the real line, we conclude that for some color $i \in \kappa$ the preimage $A=\chi^{-1}(i)$ is not meager in $\mathbb{Z}^{\omega}$. Being a Borel subset of $\mathbb{Z}^{\omega}$, the set $A$ has the Baire property, which means that for some open subset $U \subset \mathbb{Z}^{\omega}$ the symmetric difference $A \triangle U$ is meager in $\mathbb{Z}^{\omega}$. Since $A$ is not meager, the set $U$ is not empty. Take any point $c \in U \cap C$ and observe that $V=U \cap(2 c-U)$ is an open symmetric 
neighborhood of $c$. It follows that for the set $B=A \cap(2 c-A)$ the symmetric difference $B \triangle V$ is meager. Since $V$ is not meager in $\mathbb{Z}^{\omega}$, the set $B$ is not meager and hence is unbounded in $\mathbb{Z}^{\omega}$ (since totally bounded subsets of $\mathbb{Z}^{\omega}$ are nowhere dense in $\left.\mathbb{Z}^{\omega}\right)$. Now we see that $B=A \cap(2 c-A)$ is a monochromatic unbounded subset, symmetric with respect to the point $c$, witnessing that the set $C$ is $\omega$-centerpole for Borel coloring of $\mathbb{Z}^{\omega}$.

\section{Proof of Theorem 1}

Let $k \geq 2$ be a finite cardinal number and $G$ be an abelian ILC-group with totally bounded Boolean subgroup $G$ [2] and ranks $n=r_{\mathbb{R}}(G)$ and $m=r_{\mathbb{Z}}(G)$. Let $\bar{G}$ be the completion of the group with respect to its (two-sided) uniformity.

We shall give the detailed proofs of the statements (3) and (4) of Theorem 1 holding under the additional assumption of the metrizability of the group $G$ and indicate the changes which should be made for the proof of the statements (1) and (2).

Since $c_{k}^{B}\left(\mathbb{R}^{n} \times \mathbb{Z}^{m-n}\right)<\omega$ iff $k \leq m$, the statements (3), (4) of Theorem 1 will follow as soon as we prove two inequalities:

(1) $c_{k}^{B}(G) \leq c_{k}^{B}\left(\mathbb{R}^{n} \times \mathbb{Z}^{m-n}\right)$ if $k \leq m$, and

(2) $c_{k}^{B}\left(\mathbb{R}^{n} \times \mathbb{Z}^{m-n}\right) \leq c_{k}^{B}(G)$ if $c_{k}^{B}(G)$ is finite.

1. Assume that $k \leq m$. If the $\mathbb{Z}$-rank $m=r_{\mathbb{Z}}(G)$ is finite, then so is the $\mathbb{R}$-rank $n=r_{\mathbb{R}}(G)$ and we can find copies of the topological groups $\mathbb{R}^{n}$ and $\mathbb{Z}^{m}$ in $G$. Now consider the closure $H$ of the subgroup $\mathbb{R}^{n}+\mathbb{Z}^{m}$ in $G$. Since $G$ is an ILC-group and $\mathbb{R}^{n}+\mathbb{Z}^{m}$ contains a dense finitely generated subgroup, the group $H$ is locally compact. By the structure theorem of locally compact abelian groups [10, Theorem 25], $H$ is topologically isomorphic to $\mathbb{R}^{r} \oplus Z$ for some $r \in \omega$ and a closed subgroup $Z \subset H$ that contains an open compact subgroup $K$. It follows from the inclusion $\mathbb{R}^{n} \subset H$ that $n \leq r$. On the other hand, $r \leq r_{\mathbb{Z}}(G)=n$. By the same reason, $r_{\mathbb{Z}}(H)=$ $m=r_{\mathbb{Z}}(G)$. In particular, $r_{\mathbb{Z}}(Z)=m-n$ and hence $H$ contains an isomorphic copy of the group $\mathbb{R}^{n} \times \mathbb{Z}^{m-n}$. Now we see that $r_{k}^{B}(G) \leq r_{k}^{B}\left(\mathbb{R}^{n} \times \mathbb{Z}^{m-n}\right)$.

Next, assume that the $\mathbb{Z}$-rank $m=r_{\mathbb{Z}}(G)$ is infinite but $n=r_{\mathbb{R}}(G)$ is finite. By the Stabilization Lemma 11, $c_{k}^{B}\left(\mathbb{R}^{n} \times \mathbb{Z}^{m-n}\right)=c_{k}^{B}\left(\mathbb{R}^{n} \times \mathbb{Z}^{\omega}\right)=c_{k}^{B}\left(\mathbb{R}^{n} \times \mathbb{Z}^{r-n}\right)$ for $r=r c_{k}^{B}\left(\mathbb{R}^{n} \times \mathbb{Z}^{\omega}\right) \leq c_{k}^{B}\left(\mathbb{R}^{n} \times \mathbb{Z}^{\omega}\right)<\infty$. Repeating the above argument we can find a copy of the group $\mathbb{R}^{n} \oplus \mathbb{Z}^{s-n}$ in $G$ for some finite $s \geq r$ and conclude that $c_{k}^{B}(G) \leq c_{k}^{B}\left(\mathbb{R}^{n} \times \mathbb{Z}^{s-n}\right) \leq c_{k}^{B}\left(\mathbb{R}^{n} \times \mathbb{Z}^{r-s}\right)=c_{k}^{B}\left(\mathbb{R}^{n} \times \mathbb{Z}^{m-n}\right)$.

Finally, assume that the $\mathbb{R}$-rank $n=r_{\mathbb{R}}(G)$ is infinite. Then $c_{k}^{B}\left(\mathbb{R}^{n} \times \mathbb{Z}^{m-n}\right)=$ $c_{k}^{B}\left(\mathbb{R}^{\omega}\right)=c_{k}^{B}\left(\mathbb{R}^{r}\right)$ for $r=r c_{k}^{B}\left(\mathbb{R}^{\omega}\right) \leq c_{k}^{B}\left(\mathbb{R}^{\omega}\right)<\omega$. By the definition of the $\mathbb{R}$ rank $r_{\mathbb{R}}(G)=n=\omega$, we can find a copy of the group $\mathbb{R}^{r}$ in $G$ and conclude that $c_{k}^{B}(G) \leq c_{k}^{B}\left(\mathbb{R}^{r}\right)=c_{k}^{B}\left(\mathbb{R}^{n} \times \mathbb{Z}^{m-n}\right)$. This completes the proof of the inequality $c_{k}^{B}(G) \leq c_{k}^{B}\left(\mathbb{R}^{n} \times \mathbb{Z}^{m-n}\right)$.

Deleting the adjective "Borel" from the above proof we get the proof of the inequality $c_{k}(G) \leq c_{k}\left(\mathbb{R}^{n} \times \mathbb{Z}^{m-n}\right)$ holding for each $k \leq m$.

2. Now assuming that $c_{k}^{B}(G)$ is finite and the group $G$ is metrizable, we prove the inequality $c_{k}^{B}\left(\mathbb{R}^{n} \times \mathbb{Z}^{m-n}\right) \leq c_{k}^{B}(G)$. 
Fix a $k$-centerpole subset $C \subset G$ for Borel colorings of $G$ with cardinality $|C|=$ $c_{k}^{B}(G)$. The subgroup $G[2]$ is totally bounded and hence has compact closure $K_{2}$ in the completion $\bar{G}$ of the group $G$. It follows that $K_{2} \subset \bar{G}$ [2]. Since $G$ is an ILC-group, the finitely generated subgroup $\langle C\rangle$ has locally compact closure $\overline{\langle C\rangle}$ in $G$. It follows from the compactness of the subgroup $K_{2}$ that the sum $H=\overline{\langle C\rangle}+K_{2}$ is a locally compact subgroup of $\bar{G}$. This subgroup is compactly generated because it contains a dense subgroup generated by the compact set $C+K_{2}$.

By the Structure Theorem for compactly generated locally compact abelian groups [10, Theorem 24], $H$ is topologically isomorphic to $\mathbb{R}^{r} \oplus \mathbb{Z}^{s-r} \oplus K$ for some compact subgroup $K$ that contains all torsion elements of $H$. In particular, $K_{2} \subset K$. Now we see that the subgroup $2 H=\{2 x: x \in H\}$ is closed in $H$ and consequently, the subgroup $2 H \cap G$ is closed in $G$. The group $G$ is metrizable and so is the quotient group $G / 2 H$. Then the subspace $X=(G / 2 H) \backslash(H / 2 H)$ is metrizable and thus paracompact. Since $H \supset G[2]$ we can apply Lemma 9 and conclude that the set $C$ is $k$-centerpole for Borel colorings of the subgroup $H \cap G$. Since $H \cap G \subset H$, the set $C$ is $k$-centerpole for Borel colorings of the group $H$.

The compactness of the subgroup $K \subset H$ implies that the image $q(C)$ of $C$ under the quotient map $q: H \rightarrow H / K$ is a $k$-centerpole set for Borel colorings of the quotient group $H / K=\mathbb{R}^{r} \times \mathbb{Z}^{s-r}$. Since $H=\overline{\langle C\rangle}+K_{2}$ and $K_{2} \subset K$, we conclude that $\overline{\langle C\rangle} /(\overline{\langle C\rangle} \cap K)=q(\overline{\langle C\rangle})=H / K=\mathbb{R}^{r} \times \mathbb{Z}^{s-r}$ and hence $r \leq n$ and $s \leq m$. Consequently, $\mathbb{R}^{r} \times \mathbb{Z}^{s-r} \hookrightarrow \mathbb{R}^{n} \times \mathbb{Z}^{m-n}$ and

$$
c_{k}^{B}\left(\mathbb{R}^{n} \times \mathbb{Z}^{m-n}\right) \subset c_{k}^{B}\left(\mathbb{R}^{r} \times \mathbb{Z}^{s-r}\right)=c_{k}^{B}(H / K) \leq|C|=c_{k}^{B}(G) .
$$

This proves the statements (3) and (4) of Theorem 1. Deleting the adjective "Borel" from the above proof and applying Lemma 8 instead of Lemma 9, we get the proof of the inequality $c_{k}\left(\mathbb{R}^{n} \times \mathbb{Z}^{m-n}\right) \leq c_{k}(G)$ under the assumption that the number $c_{k}(G)$ is finite. Since Lemma 8 does not require the metrizability of $G$, this upper bound holds without this assumption. In such a way, we prove the statements (1) and (2) of Theorem 1.

\section{Proof of Proposition 1}

Let $G$ be a metrizable abelian ILC-group with totally bounded Boolean subgroup $G$ [2] and $k \in \mathbb{N}$ be such that $2 \leq k \leq r_{\mathbb{Z}}(G)$. Theorems 1 and 3 guarantee that $c_{k}^{B}(G)=c_{k}^{B}\left(\mathbb{R}^{n} \times \mathbb{Z}^{m-n}\right)<\infty$ where $n=r_{\mathbb{Z}}(G)$ and $m=r_{\mathbb{Z}}(G)$.

Let $r=r c_{k}(G)$ and $C \subset G$ be a subset of cardinality $|C|=c_{k}^{B}(G)$ such that $r_{\mathbb{Z}}(\langle C\rangle)=r$. Without loss of generality, $0 \in C$. Since $G$ is an ILC-group, the finitely generated subgroup $\langle C\rangle$ has locally compact closure in $G$.

The totally bounded Boolean subgroup $G$ [2] has compact closure $K_{2}$ in the completion $\bar{G}$ of the abelian topological group $G$. It follows that the subgroup $H=\overline{\langle C\rangle}+K_{2}$ of $\bar{G}$ is locally compact and compactly generated. Consequently, it contains a compact subgroup $K \supset K_{2}$ such that the quotient group $H$ / $K$ is topologically isomorphic to $\mathbb{R}^{s} \times \mathbb{Z}^{r-s}$ for some $r \leq s$. It follows from Lemma 8 that the set $C$ is $k$-centerpole for Borel colorings of the group $H$. The compactness of the subgroup 
$K \subset H$ implies that the image $q(C) \subset H / K$ of $C$ under the quotient homomorphism $q: H \rightarrow H / K$ is a $k$-centerpole set for Borel colorings of $H / K$. Consequently,

$$
c_{k}^{B}\left(\mathbb{R}^{r}\right) \leq c_{k}^{B}\left(\mathbb{R}^{s} \times \mathbb{Z}^{r-s}\right)=c_{k}^{B}(H / K) \leq|q(C)| \leq|C|=c_{k}^{B}(G)<\infty
$$

and hence $r \geq k$ by Theorem 3(5).

Now assume that $k \geq 4$. Since the set $q(C)$ is $k$-centerpole for Borel colorings of $H / K=\mathbb{R}^{s} \times \mathbb{Z}^{r-s} \subset \mathbb{R}^{r}$, Lemma 10 implies that the affine hull of $q(C)$ in the linear space $\mathbb{R}^{r}$ has dimension $\leq|q(C)|-3$. Since $0 \in q(C)$, the affine hull of the set $q(C)$ coincides with its linear hull. Consequently, $r=r_{\mathbb{Z}}(\langle C\rangle)=r_{\mathbb{Z}}(\langle q(C)\rangle) \leq$ $|q(C)|-3 \leq|C|-3=c_{k}^{B}(G)-3$. This completes the proof of the lower and upper bounds

$$
k \leq r c_{k}(G) \leq c_{k}^{B}(G)-3
$$

for all $k \geq 3$.

Next, we show that $r c_{k}(G)=k$ for $k \in\{2,3\}$. In this case $c_{k}^{B}(G)=c_{k}\left(\mathbb{Z}^{k}\right)$ by Theorems 1 and 2. Since $r_{\mathbb{Z}}(G) \geq k$, the group $G$ contains an isomorphic copy of the group $\mathbb{Z}^{k}$. Then each $k$-centerpole subset $C \subset \mathbb{Z}^{k} \subset G$ with $|C|=c_{k}\left(\mathbb{Z}^{k}\right)$ is $k$ centerpole for Borel colorings of $G$ and thus $k \leq r c_{k}^{B}(G) \leq r_{\mathbb{Z}}(\langle C\rangle) \leq k$, which implies the desired equality $r c_{k}^{B}(G)=k$.

\section{Proof of Stabilization Theorem 4}

Let $k \geq 2$ and $G$ be an abelian ILC-group with totally bounded Boolean subgroup $G[2]$. Let $n=r_{\mathbb{R}}(G)$ and $m=r_{\mathbb{Z}}(G)$.

1. Assume that $m=r_{\mathbb{Z}}(G) \geq r c_{k}^{B}\left(\mathbb{Z}^{\omega}\right)$. By Proposition $1, k \leq r c_{k}^{B}\left(\mathbb{Z}^{\omega}\right) \leq r_{\mathbb{Z}}(G)$ and then $c_{k}(G)=c_{k}\left(\mathbb{R}^{n} \times \mathbb{Z}^{m-n}\right)$ by Theorem 1 . Since $m=r_{\mathbb{Z}}\left(\mathbb{R}^{n} \times \mathbb{Z}^{m-n}\right) \geq$ $r c_{k}^{B}\left(\mathbb{Z}^{\omega}\right)$, Lemma 12 guarantees that $c_{k}(G)=c_{k}^{B}\left(\mathbb{R}^{n} \times \mathbb{Z}^{m-n}\right)=c_{k}^{B}\left(\mathbb{Z}^{\omega}\right)$.

2. Assume that the group $G$ is metrizable and $r_{\mathbb{Z}}(G) \geq r c_{k}^{B}\left(\mathbb{R}^{n} \times \mathbb{Z}^{\omega}\right)$. By Proposition $1, k \leq r c_{k}^{B}\left(\mathbb{R}^{n} \times \mathbb{Z}^{\omega}\right) \leq r_{\mathbb{Z}}(G)=m$ and hence $c_{k}^{B}(G)=c_{k}^{B}\left(\mathbb{R}^{n} \times \mathbb{Z}^{m-n}\right)$ by Theorem 1. Since $m=r_{\mathbb{Z}}\left(\mathbb{R}^{n} \times \mathbb{Z}^{m-n}\right) \geq r c_{k}^{B}\left(\mathbb{R}^{n} \times \mathbb{Z}^{\omega}\right)$, Lemma 11 guarantees that $c_{k}^{B}(G)=c_{\mathbb{Z}}^{B}\left(\mathbb{R}^{n} \times \mathbb{Z}^{m-n}\right)=c_{k}^{B}\left(\mathbb{R}^{n} \times \mathbb{Z}^{\omega}\right)$.

3. By analogy with the preceding case we can prove that $c_{k}^{B}(G)=c_{k}^{B}\left(\mathbb{R}^{\omega}\right)$ if $G$ is metrizable and $r_{\mathbb{R}}(G) \geq r c_{k}^{B}\left(\mathbb{R}^{\omega}\right)$.

\section{Selected open problems}

By Theorem $2, c_{k}^{B}\left(\mathbb{R}^{\omega}\right)=c_{k}\left(\mathbb{Z}^{\omega}\right)=c_{k}\left(\mathbb{Z}^{k}\right)$ for all $k \leq 4$.

Problem 1 Is $c_{k}\left(\mathbb{Z}^{\omega}\right)=c_{k}\left(\mathbb{Z}^{k}\right)$ for all $k \in \mathbb{N}$ ? In particular, is $c_{4}\left(\mathbb{Z}^{n}\right)=12$ for every $n \geq 4$ ?

Problem 2 Is $c_{k}^{B}\left(\mathbb{R}^{n}\right)=c_{k}\left(\mathbb{R}^{n}\right)$ for every $k \leq n$ ? 
Theorem 3 gives upper and lower bounds for the numbers $c_{k}\left(\mathbb{Z}^{k}\right)$ that have exponential and polynomial growths, respectively.

Problem 3 Is the growth of the sequence $\left(c_{n}\left(\mathbb{Z}^{n}\right)\right)_{n \in \mathbb{N}}$ exponential?

By [1], for every $k \in\{1,2,3\}$ any $k$-centerpole subset $C \subset \mathbb{Z}^{k}$ of cardinality $|C|=$ $c_{k}\left(\mathbb{Z}^{k}\right)$ is affinely equivalent to the $\left(\begin{array}{l}k-1 \\ k-3\end{array}\right)$-sandwich $\Xi_{k-3}^{k-1}$.

Problem 4 Is each 12-element 4-centerpole subset of $\mathbb{Z}^{4}$ affinely equivalent to the $\left(\begin{array}{l}3 \\ 1\end{array}\right)$-sandwich $\Xi_{1}^{3}$ ?

It follows from the proof of Theorem 1 in [8] that the free group $F_{2}$ with two generators and discrete topology has $c_{2}\left(F_{2}\right) \leq 13$.

Problem 5 What is the value of the cardinal $c_{2}\left(F_{2}\right)$ ? Is $c_{3}\left(F_{2}\right)$ finite?

The last problem can be posed in a more general context.

Problem 6 Investigate the cardinal characteristics $c_{k}(G)$ and $c_{k}^{B}(G)$ for noncommutative topological groups $G$.

\section{References}

1. Banakh, T.: On a cardinal group invariant related to partition of abelian groups. Mat. Zametki 64(3), 341-350 (1998)

2. Banakh, T., Bokalo, B., Guran, I., Radul, T., Zarichnyi, M.: Problems from the Lviv topological seminar. In: Pearl, E. (ed.) Open Problems in Topology, vol. II, pp. 655-667. Elsevier, Amsterdam (2007)

3. Banakh, T., Dudko, A., Repovs, D.: Symmetric monochromatic subsets in colorings of the Lobachevsky plane. Discrete Math. Theor. Comput. Sci. 12(1), 12-20 (2010)

4. Banakh, T., Protasov, I.V.: Asymmetric partitions of Abelian groups. Mat. Zametki 66(1), 17-30 (1999)

5. Banakh, T., Protasov, I.: Symmetry and colorings: some results and open problems. Izv. Gomel Univ. Vopr. Algebry 4(17), 5-16 (2001)

6. Banakh, T., Verbitski, O., Vorobets, Ya.: Ramsey treatment of symmetry. Electron. J. Comb. 7(1), R52 (2000), 25 pp.

7. Engelking, R.: General topology. Sigma Series in Pure Mathematics, vol. 6. Heldermann Verlag, Berlin (1989)

8. Gryshko, Yu.: Monochrome symmetric subsets in 2-colorings of groups. Electron. J. Comb. 10, R28 (2003), 8 pp.

9. Just, W., Weese, M.: Discovering Modern Set Theory. II. Set-Theoretic Tools for Every Mathematician. GSM, vol. 18. Am. Math. Soc., Providence (1997)

10. Morris, S.: Pontryagin Duality and the Structure of Locally Compact Abelian Groups. London Mathematical Society Lecture Note Series, vol. 29. Cambridge University Press, Cambridge (1977)

11. Protasov, I.V.: Some problems in combinatorics of colorings. U Svìtì Mat. 1, 61 (1995) (in Ukrainian)

12. Protasov, I.V.: Asymmetrically decomposable abelian groups. Mat. Zametki 59(3), 468-471 (1996)

13. Spanier, E.H.: Algebraic Topology. McGraw-Hill, New York (1966) 